\title{
27. PLEISTOCENE PLANKTONIC FORAMINIFERAL ASSEMBLAGES AND PALEOTEMPERATURE FLUCTUATIONS IN JAPAN SEA, SITE 798 1
}

\author{
Tara Kheradyar $^{2}$
}

\begin{abstract}
ODP Site 798 on the Oki Ridge in the Southern Japan Sea yielded the first continuous and well-preserved record of Pleistocene planktonic foraminifers in the Northwestern Pacific Ocean region. Quantitative analysis of planktonic foraminifers completed for 122 samples from the 200-m-thick Pleistocene section cored at ODP Site 798 provides a proxy record of variations in sea-surface temperature, productivity, and circulation during the past $1.6 \mathrm{~m} . \mathrm{y}$. in an area beneath the track of the Tsushima Current. Faunal census data allow recognition of five distinct assemblages: (1) type A assemblages dominated by sinistrally coiling forms of Neogloboquadrina pachyderma representing polar-subpolar surface temperatures, 2) type B assemblages dominated by Globigerina bulloides and thought to represent periods of increased surface productivity and upwelling, (3) type C assemblages marked by significant abundances of dextrally coiling forms of $N$. pachyderma thought to represent the warm transitional waters of the Tsushima Current, (4) type D assemblages distinguished by relatively high percentages of dextral $N$. pachyderma and Globorotalia inflata that also represent warmer surface temperatures and increased flow of the Tsushima Current, and (5) type $\mathrm{E}$ assemblages marked by relatively large numbers of the delicate species Globigerina quinqueloba and Globigerinita spp., indicative of exceptional preservation conditions and/or episodic high production of these taxa.

Early and middle Pleistocene coiling patterns of Neogloboquadrina pachyderma at Site 798 can be correlated with Pleistocene coiling trends and planktonic foraminiferal datums established in the onshore Oga Peninsula sequence of Northern Honshu and open-ocean $N$. pachyderma coiling dominance shifts in the North Pacific region. A sustained early Pleistocene warm period recognized in both the Oga Peninsula sequence and the Northern Pacific can clearly be recognized at Site 798. In addition, the late Pleistocene planktonic foraminiferal record at Site 798 shows good correlation with glaciation/deglaciation events for the Northern Hemisphere as delineated by oxygen isotopes and represents the first detailed analysis of Pleistocene sea-surface temperature changes in the Northwestern Pacific Ocean region.
\end{abstract}

\section{INTRODUCTION}

The majority of the information on Quaternary global climatic fluctuations is based on evidence from open-ocean sequences (e.g., Shackleton and Opdyke, 1977; Savin, 1977; Imbrie et al., 1984) or from terrestrial settings (e.g., glacier advance/retreat; Broecker and Denton, 1989). By contrast, marginal basins such as the Japan Sea have been little studied despite their sensitivity to glacial/interglacial sea-level changes. The lack of a detailed assessment of the sedimentary history of the Japan Sea can be partially attributed to poor sediment recovery prior to the advent of hydraulic piston coring. In addition, poor carbonate preservation at the sites drilled during Deep Sea Drilling Project (DSDP) Leg 31 in 1973 yielded dissolved foraminiferal assemblages (Karig, Ingle, et al., 1975; Fig. 1). However, more recent drilling during Ocean Drilling Program (ODP) Legs 127 and 128 recovered continuous sequences of Pleistocene carbonate-rich sediments from several sites in the southern region of the Japan Sea (Tamaki, Pisciotto, Allan, et al., 1990; Ingle, Suyehiro, von Breymann, et al., 1990). These latter sediments provide exceptional opportunities for comprehensive studies of Pleistocene climatic fluctuations in the Japan Sea region and permit comparison with changes analyzed in the adjacent Pacific Ocean. Planktonic foraminifers are exceptionally well preserved at ODP Site 798 on the Oki Ridge (Fig. 1) and form the basis for this report. Pleistocene planktonic foraminiferal assemblages from Site 798 were analyzed quantitatively and used to interpret variations in surface circulation and sea-surface temperatures in the Japan Sea and, in turn, were compared to Pleistocene planktonic foraminiferal trends established in the open Pacific and variations in global climate inferred from oxygen isotopes.

\footnotetext{
${ }^{1}$ Pisciotto, K. A., Ingle, J. C., Jr., von Breymann, M. T., Barron, J., et al., 1992. Proc. $O D P$, Sci. Results, 127/128, Pt. 1: College Station, TX (Ocean Drilling Program).

${ }^{2}$ Geology Department, Stanford University, Stanford, CA $94305-2115$, U.S.A.
}

\section{Hydrography of the Japan Sea}

The origin of the Japan Sea and its oceanographic history have been interpreted through studies of deep-sea cores and analysis of Neogene marine sections exposed on the Japanese Islands (Matoba, 1984). Deep-sea sediments have been recovered by conventional piston coring (e.g., Ujiie and Ichikura, 1973), by rotary drilling during Leg 31 (Karig, Ingle, et al., 1975), and by hydraulic piston coring during Legs 127 (Tamaki, Pisciotto, Allan, et al., 1990) and 128 (Ingle, Suyehiro, von Breymann, et al., 1990). Prior to Legs 127 and 128 , geophysical evidence had prompted the suggestion that the Japan Sea began to open in the middle to early Miocene (Tamaki, 1986); a prediction that was confirmed by the recovery of lower Miocene sediments during ODP drilling. Significantly, the Japan Sea became increasingly isolated from the Pacific Ocean as a result of tectonic uplift and deformation during late Pliocene and Pleistocene time, creating the modern backarc marginal sea connected to Pacific Ocean surface waters through four shallow straits (Matoba, 1984; Fig. 1).

At present, the cold surface waters of the Liman Current originate in the Mamiya (or Tartar) Strait (sill depth $12 \mathrm{~m}$ ) in the Northern Japan Sea; to the east, the sea is in contact with the cold North Pacific Oyashio Current through the Soya $(55 \mathrm{~m})$ and Tsugaru $(130 \mathrm{~m})$ straits. To the south the Tsushima Current, a branch of the warm Kuroshio Current, enters the sea via the Tsushima Strait (140 m). Circulation in the Japan Sea involves discrete surface- and deep-water masses that are highly sensitive to climate change and were subject to rapid changes throughout the glacial/interglacial cycles of the Quaternary (Matoba, 1984; Oba et al., 1991). During interglacial periods the Tsushima Current readily enters the sea because of associated high stands of sea level and greater depth of water in the Tsushima Strait. Seasonal melting of sea ice in the northern portion of the modern sea generates cold, dense, high-salinity waters that sink to form the unusually cold Japan Sea intermediate and bottom waters and ventilate the entire sea in the process. Matoba (1984) suggested that as a result of the well-mixed character of the water column in the Japan 


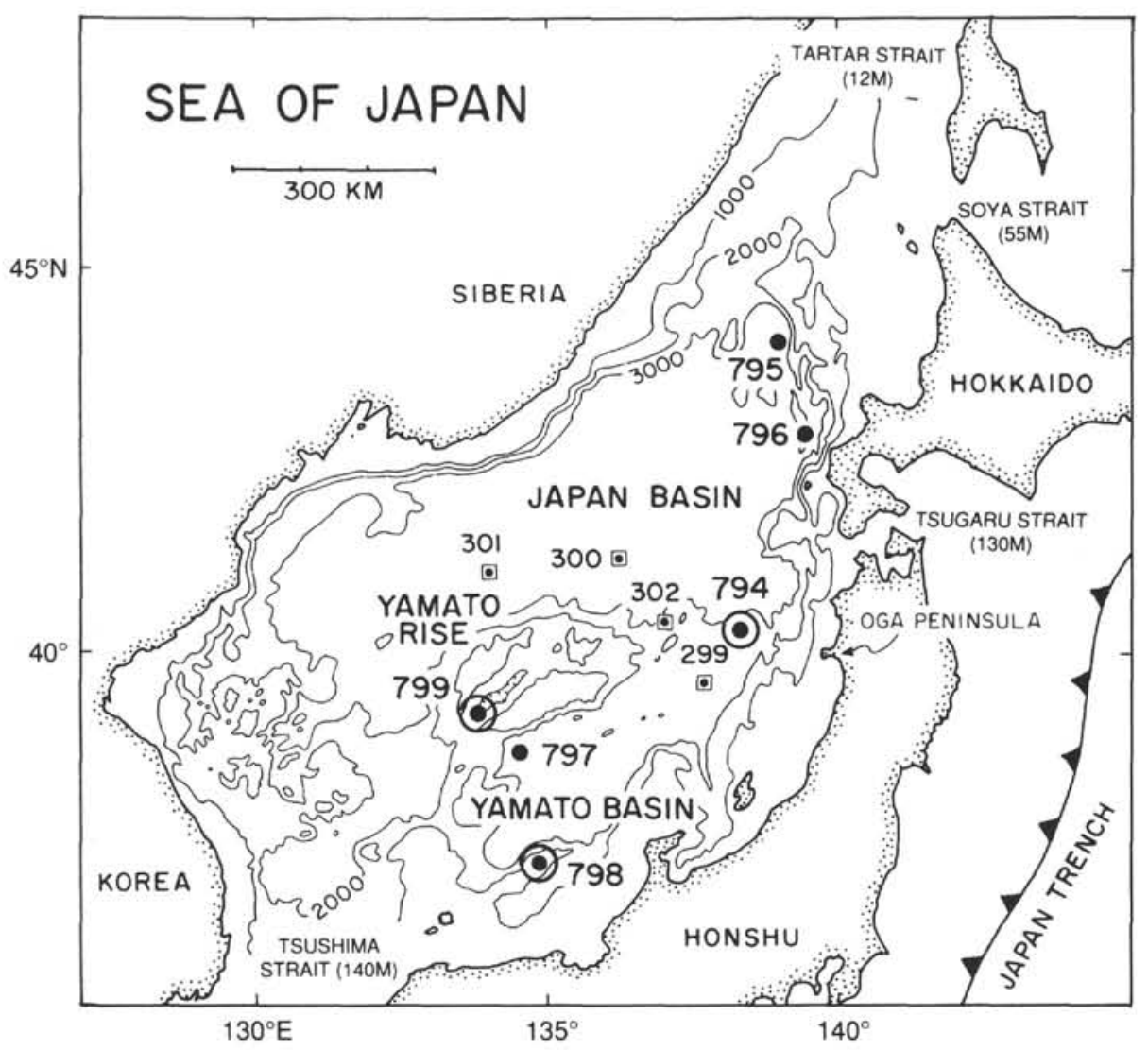

Figure 1. Location map of the Japan Sea showing ODP Leg 128 Sites 794, 798, and 799 (circled dots), Leg 127 Sites 794 through 799 (dots), DSDP Sites 299 through 302 (boxed dots), the four major straits connecting the sea to the Pacific Ocean, and the Oga Peninsula onshore Neogene reference section. Note that Site 794 was occupied during both Legs 127 and 128. Bathymetry in meters.

Sea the depth of the local carbonate compensation depth (CCD) is unusually shallow $(2000 \mathrm{~m})$ in comparison with that of the open Pacific Ocean. In the central portion of the sea, warm Tsushima surface water meets cold surface water from the north creating an important oceanographic front. Weather fronts with turbulent air masses may also have formed at this location during interglacials, giving rise to generally very wet (monsoonal) periods (Meyers et al., in press, and references therein). These conditions were reversed during glacial periods when the Japan Sea became semi-isolated due to the global fall in sea level and restrictions of warm water from the south. It has also been suggested (Oba et al., 1991) that fresh water from the Yellow River entered the sea during some glacial intervals. Less vigorous mixing of the water masses during glacial intervals when drier weather systems become dominant possibly created dysaerobic conditions in the bottom waters of the Japan Sea.

Detailed records of Quaternary glacial/interglacial events within the Japan Sea are not well described despite the importance of the sea as an amplified monitor of climate changes in the Northeast Pacific region, with the notable exception of a recent report by Oba et al. (1991) detailing events over the last few thousand years. Fortunately, Legs 127 and 128 (Tamaki, Pisciotto, Allan, et al., 1990; Ingle, Suyehiro, von Breymann, et al., 1990) recovered well-preserved cyclic sequences of Quaternary sediments that clearly record major changes in productivity, dissolved oxygen, and water circulation within the sea over the past 2 m.y. These latter sediments appear as remarkable interbedded sequences of dark, organic-rich layers and lighter, less organic-rich bioturbated intervals which likely formed as sedimentary responses to high-frequency oscillations in the oceanog- raphy of the basin. The light-dark cycles are best developed at Site 798 on Oki Ridge (Fig. 1). Overall, these sediments offer a unique opportunity to decipher the Pleistocene paleoceanographic history of the Japan Sea. In addition, the sensitivity of the sea to eustatic events makes it an ideal setting for the exploration of past climatic fluctuations on a regional scale, which bears on the relationship between sea-surface temperature and ice volume in the global oceanic record.

\section{Site 798 (Oki Ridge)}

ODP Site 798 is located on Oki Ridge (Fig. 1) at a depth of $900 \mathrm{~m}$ (Ingle, Suyehiro, von Breymann, et al., 1990). This site was selected specifically to obtain a paleoceanographic reference section deposited above the local CCD and to maximize recovery of carbonate-bearing sediments (i.e., sediments rich in planktonic foraminifers) which would allow detailed study of surface circulation changes in the Japan Sea. As expected, the sediments recovered include a continuous record of planktonic foraminifers through the Pleistocene, as well as an impressive series of decimeter- to meter-scale depositional cycles involving the repetition of dark-laminated and light-bioturbated subunits noted previously. Approximately $570 \mathrm{~m}$ of Pliocene and Pleistocene sediments was recovered at this site before safety concerns over increased levels of ethane and propane caused drilling to be stopped. Microfossil and paleomagnetic stratigraphy at Site 798 revealed that Pleistocene sediments are present to a depth of $214 \mathrm{~m}$ below seafloor (mbsf) (Ingle, Suyehiro, von Breymann, et al., 1990). The sediment-accumulation rate at this site over the past $2.5 \mathrm{~m}$.y. has remained fairly constant, averaging approximately $12 \mathrm{~cm} / \mathrm{k}$.y., a value 
that is higher than typical pelagic sedimentation in the open oceans. The comparatively high sedimentation rate has enhanced the preservation of calcareous microfossils.

\section{Previous Studies of Quaternary Paleoclimate in the Northern Pacific Ocean and Japan Sea Region}

Fluctuations of Pleistocene sea-surface temperatures in the Japan Sea have been in part dictated by changes in surface circulation induced by sea-level changes, associated with polar ice accumulation as well as southward advances of the Polar Front in the Northwestern Pacific Ocean. Specifically, formation of continental ice sheets and lowering of the sea level during glacial maxima periodically led to the reduction of inflow of the Tsushima Current into the sea and, in turn, to the exclusion of transitional to tropical planktonic foraminifers. It is useful to review some of the previous studies of sea-surface temperature fluctuations in the Northern Pacific Ocean and the Japan Sea region prior to describing the Pleistocene sea-surface temperature changes detected at Site 798.

Several studies have utilized planktonic foraminifers, especially variations in the coiling ratio of Neogloboquadrina pachyderma, to identify paleoclimatic signals in the mid-latitude $\left(35^{\circ}-45^{\circ} \mathrm{N}\right)$ regions of the Northern Pacific Ocean (Olsson and Gull, 1970; Olsson, 1974; Ingle, 1973a, 1973b; Echols, 1973; Vincent, 1975; Keller, 1978, 1980; Butt, 1980; Thompson, 1980; Lagoe, 1986). These studies had variable success depending on the degree of dissolution and diversity of foraminifers. Olsson and Gull (1970) and Ingle, (1973a, 1973b) documented relatively warm early Pleistocene sea-surface temperatures in the Northeast Pacific. Ingle (1973a) reported an onset of cooler and fluctuating surface conditions at about 1.2 Ma at DSDP Site 173 off California, an event recognized earlier by Kent et al. (1971), based on analysis of ice-rafted sediments. Olsson (1974) recognized seven warm/cold intervals in the Brunhes Magnetochron that are similar to the ice-rafting curve of Kent et al. (1971). In the North-Central Pacific region, Vincent (1975) and Keller (1978) recognized three and four warm intervals, respectively, inundated with cooler events during the late and early Pleistocene. Studies of faunas from DSDP sites in the Northwestern Pacific and the Japan Trench areas by Thompson (1980) and Keller (1980) recognized warmer conditions in the early Pleistocene based on coiling ratio changes of $N$. pachyderma. Although dissolution was recognized to be significant in the Japan Trench sites, Thompson (1980) was able to identify six warm/cold intervals within the Brunhes Magnetochron.

In addition to studies of deep-sea cores, the Pleistocene paleoenvironment in the Japan Sea has also been investigated through analysis of planktonic foraminifers from onshore marine sections exposed in northwestern Honshu. Matoba et al. (1990) summarized the magnetostratigraphic and biostratigraphic data and correlations in the Oga Peninsula section, which is the most important Neogene reference section for Northwestern Japan, including the Akita and Niigata oil-producing regions (Fig. 2). Three planktonic foraminifer zones span the Pleistocene in the Oga Peninsula (Maiya, 1975, 1978; Maiya et al., 1976). Early Pleistocene Zone 7 (Neogloboquadrina pachyderma dextral/Globorotalia orientalis Zone) spans most of the Kitaura Formation. Foraminiferal Zone 7 is separated from Zones 8 and 9 at a major shift from dominantly dextrally coiling to dominantly sinistrally coiling populations of $N$. pachyderma. This coiling shift apparently corresponds to the onset of mid-latitude glaciation reported by Kent et al. (1971) in the Northern Pacific Ocean. The uppermost Kitaura and overlying Wakimoto and Shibikawa Formations are within foraminiferal Zones 8 and $9(N$. pachyderma sinistral/G. quinqueloba and $N$. pachyderma sinistral/Globigerina incompta Concurrent Range Zones). Two shorter shifts in coiling dominance of $N$. pachyderma occur within Zones 8 and 9 and most likely correlate with two of the numerous warming events recorded by Kent et al. (1971) in the Brunhes Magnetochron.
In the Japan Sea proper, Ingle (1975) examined the stratigraphic variation of planktonic foraminifers from DSDP Site 299 and recognized several episodes of sea-surface temperature fluctuation in the Pleistocene. In particular, the initiation of severe mid-latitude glaciation at $0.9 \mathrm{Ma}$ following the onset of mid-latitude glaciation at $1.2 \mathrm{Ma}$ (Kent et al., 1971) is clearly expressed at Site 299. In addition, Ingle reported a warming episode at about $0.5 \mathrm{Ma}$ related to the climatic changes in the Northwestern Pacific. However, the numerous episodes of ice rafting associated with the glaciation/deglaciation events of the Brunhes Magnetochron in the Northwestern Pacific of Kent et al. (1971) have not been fully recognized in the Japan Sea region or the land-based marine section of Japan.

Thus, earlier studies illustrate that planktonic foraminiferal evidence of the major Pleistocene climatic fluctuations in the Northern Pacific Ocean is present in the Japan Sea and that the recognition of global events in the Japan Sea record is possible. Significantly, Lagoe and Thompson (1988) developed a regional Neogloboquadrina pachyderma coiling dominance (CD) curve for the North Pacific based on the synthesis of various studies of deep-sea cores and land-based sections (Fig. 3). The Lagoe and Thompson curve and numbered CD shifts are herein used as the Northern Pacific standard and the basis for comparison and correlation of Pleistocene planktonic foraminiferal events in the Japan Sea as analyzed at ODP Site 798 (Fig. 1).

\section{METHODS}

A total of 121 samples-109 from Hole 798A (0-146 mbsf; Cores 128-798A-1H through 128-798A-15H) and 12 from Hole $798 \mathrm{~B}$ (147-210 mbsf; Cores 128-798B-16X through 128-798B-22X) was analyzed for planktonic foraminiferal content (Table 1). The samples represent an average sample resolution of 8-20 k.y. based on biostratigraphic and magnetostratigraphic ages for the Pleistocene sequence at Site 798 (Ingle, Suyehiro, von Breymann, et al., 1990). The samples were soaked in plain water for $24 \mathrm{hr}$ and washed using a sieve with a $63-\mu \mathrm{m}$-diameter opening. Small splits of the dried residue were inspected for planktonic foraminifers and approximately 300 specimens were picked randomly and identified (Table 1); all specimens were picked from samples containing fewer than 300 individuals. Faunal census data were subsequently used to calculate the relative abundances of taxa in each sample in terms of percent of total planktonic species counted. Variations in dominant taxa allowed assemblages to be divided into several distinct types and subtypes for the purpose of paleoenvironmental interpretation (Tables 1 and 2 and Figs. 2 and 3 ).

\section{VARIATION IN FAUNAL COMPOSITION}

Planktonic foraminiferal faunal provinces have been recognized and described from the Atlantic, Indian, and Pacific oceans by many different authors using both plankton net and core-top samples (e.g., Bradshaw, 1959; Bé and Thoderlund, 1971; Imbrie and Kipp, 1971; Kipp, 1976; Thompson, 1981). These authors have identified similar low-, mid-, and high-latitude provinces within all three major ocean basins. I use the faunal associations established by Thompson (1981) for the Northwestern Pacific Ocean to describe and analyze the planktonic foraminiferal assemblages and trends at Site 798 in the Japan Sea. Thompson described six distinct and paleoceanographically sensitive faunas as follows:

1. polar-subpolar fauna dominated by sinistrally coiling Neogloboquadrina pachyderma and variable abundances of Globigerina bulloides, dextrally coiling $N$. pachyderma, and Globigerina quinqueloba;

2. transitional fauna marked by Globorotalia inflata, dextrally coiling N. pachyderma, and a few Globorotalia eggeri (= Neogloboquadrina dutertrei in this paper); 


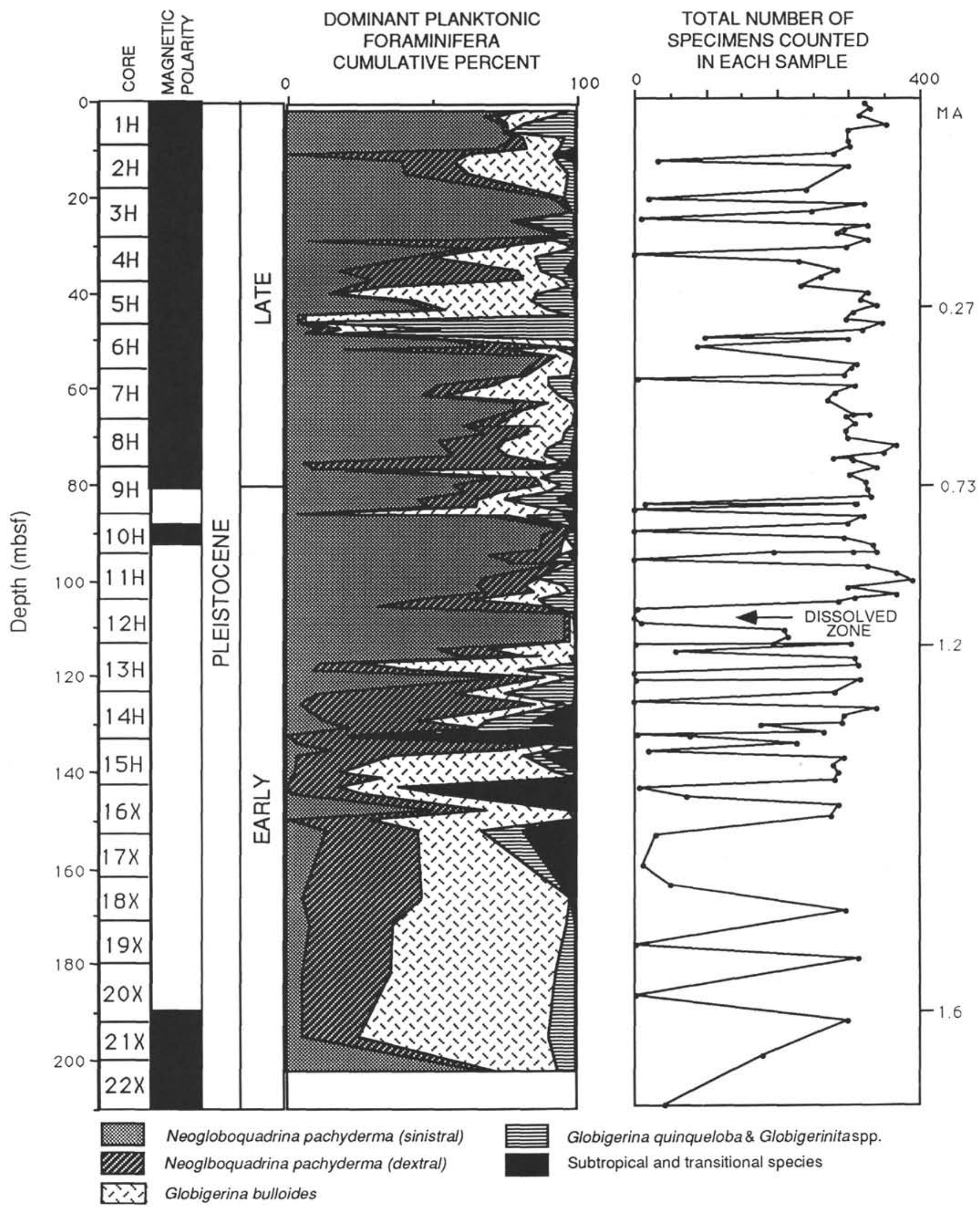

Figure 2. Cumulative curve illustrating variations in the relative percentages of the dominant species of planktonic foraminifers at Site 798 . The curve on the right shows the number of specimens counted in each sample. The samples containing fewer than 50 specimens were not used in constructing the cumulative distribution curve. The magnetostratigraphy at Site 798 is from Ingle, Suyehiro, von Breymann, et al. (1990). 
GENERALIZED COILING DIOMINANCE (CD) SHIFTS FOR THE NORTH PACIFIC

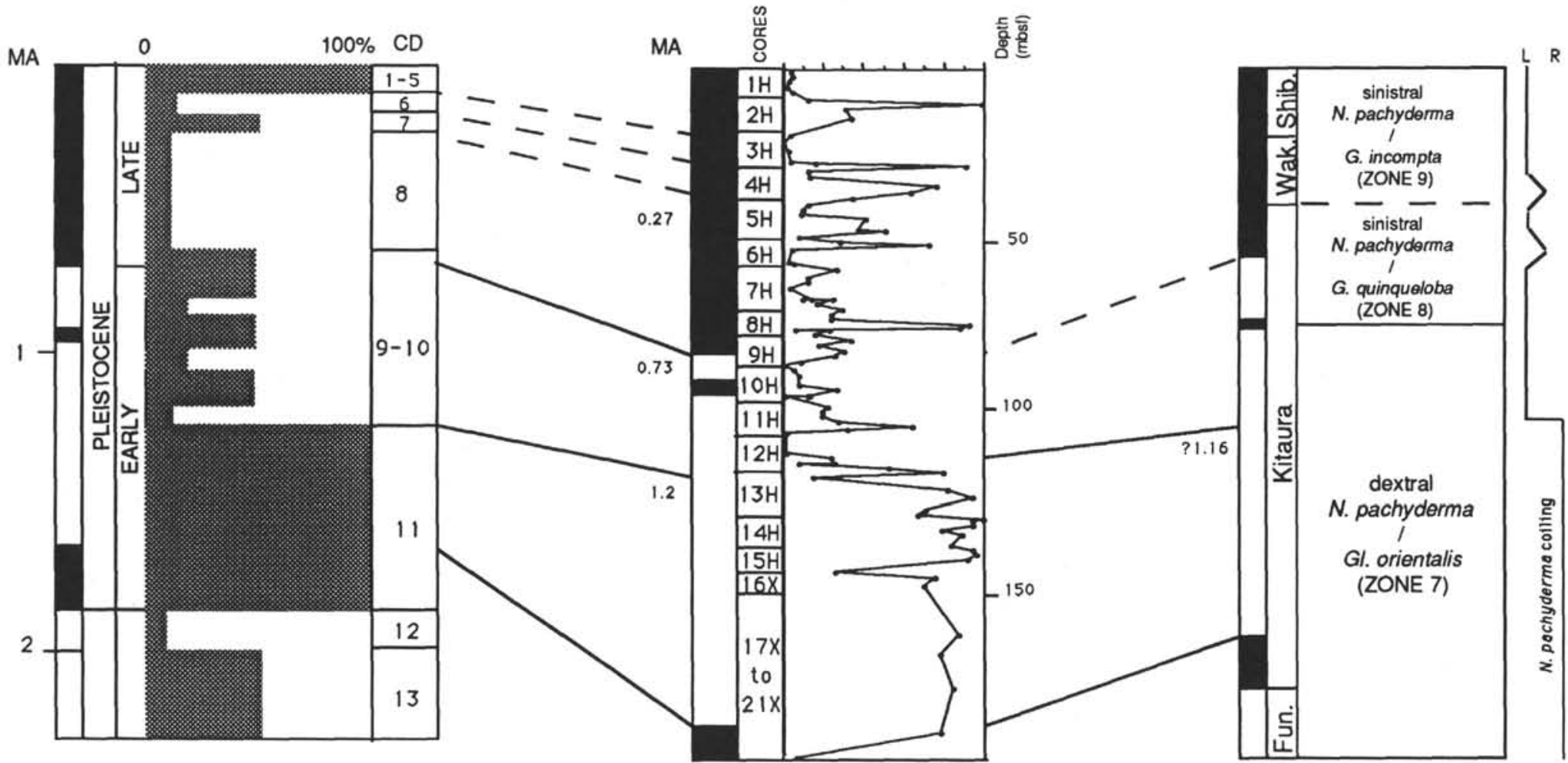

Figure 3. Site 798 Neogloboquadrina pachyderma coiling dominance (CD) curve correlated with the CD shifts of Lagoe and Thompson (1988) for the Northern Pacific Ocean and planktonic foraminifer zones, N. pachyderma coiling dominance, and magnetic polarity record for the Oga Peninsula summarized by Matoba et al. (1990). The age datums for Site 798 are from Ingle, Suyehiro, von Breymann, et al. (1990). Samples that included fewer than 20 specimens of $N$. pachyderma were not used for the construction of the coiling dominance curve. Dashed lines represent tentative correlations. Shib. $=$ Shibikawa, Wak. $=$ Wakimoto, Fun.$=$ Funukawa. 
Table 1. Pleistocene planktonic foraminiferal assemblages identified at Site 798.

\begin{tabular}{|c|c|c|c|c|c|c|c|c|c|c|c|}
\hline $\begin{array}{l}\text { Core, section. } \\
\text { interval (cm) }\end{array}$ & $\begin{array}{l}\text { Depth } \\
\text { (mbsf) }\end{array}$ & $\begin{array}{c}\text { Globigerina } \\
\text { bulloides }\end{array}$ & $\begin{array}{l}\text { Globigerina } \\
\text { bulloides } \\
\text { umbilicata }\end{array}$ & $\begin{array}{l}\text { Globigerina } \\
\text { quinqueloba }\end{array}$ & $\begin{array}{l}\text { Globigerinita } \\
\text { spp. }\end{array}$ & $\begin{array}{l}\text { Globorotalia } \\
\text { inflata }\end{array}$ & $\begin{array}{l}\text { Globorotalia } \\
\text { menandii }\end{array}$ & $\begin{array}{c}\text { Globigerinoides } \\
\text { ruber }\end{array}$ & $\begin{array}{l}\text { Globigerinoides } \\
\text { sp. }\end{array}$ & $\begin{array}{l}\text { Neogloboquadrina } \\
\text { dutertrei }\end{array}$ & $\begin{array}{l}\text { Neogloboquadrina } \\
\text { pachyderma } \\
\text { dextral }\end{array}$ \\
\hline \multicolumn{12}{|l|}{ 128-798A- } \\
\hline $1 \mathrm{H}-1,108-114$ & 1.08 & 49 & 1 & 8 & 0 & 0 & 0 & 0 & 0 & 0 & 9 \\
\hline $1 \mathrm{H}-2,77-83$ & 2.27 & 59 & 5 & 23 & 4 & 0 & 0 & 0 & 0 & 0 & 11 \\
\hline $1 \mathrm{H}-3,80-86$ & 3.8 & 20 & $i$ & 31 & 23 & 0 & 0 & 0 & 0 & 0 & 6 \\
\hline $1 \mathrm{H}-4,106-112$ & 5.56 & 0 & 0 & 61 & 20 & 0 & 0 & 0 & 0 & 0 & 4 \\
\hline IH-5. 84-90 & 6.84 & 42 & 0 & 11 & 1 & 0 & 0 & 0 & 0 & 0 & 10 \\
\hline IH-CC & 9 & 31 & 2 & is & 2 & 0 & 0 & 0 & 0 & 0 & 32 \\
\hline $2 \mathrm{H}-1.79-85$ & 10.09 & 84 & 4 & 0 & 0 & 0 & 0 & 9 & 1 & 13 & 188 \\
\hline $2 \mathrm{H}-2.79-85$ & 11.59 & 94 & 4 & 15 & i & 0 & 0 & 0 & 0 & 2 & 51 \\
\hline $2 \mathrm{H}-3,79-84$ & 13.09 & 14 & 0 & 8 & 0 & 0 & 0 & 0 & 0 & 0 & 2 \\
\hline $2 \mathrm{H}-\mathrm{CC}$ & 14.31 & 99 & 5 & 5 & 2 & 0 & 0 & 0 & 0 & 0 & 65 \\
\hline $3 \mathrm{H}-1.80-86$ & 19.1 & 1 & 0 & 9 & 0 & 0 & 0 & 0 & 0 & 0 & 9 \\
\hline $3 \mathrm{H}-2,105-111$ & 20.85 & 0 & 0 & 0 & 0 & 0 & 0 & 0 & 0 & 0 & 1 \\
\hline $3 \mathrm{H}-3,80-86$ & 22.1 & 0 & 0 & 4 & 0 & 0 & 0 & 0 & 0 & 0 & $i$ \\
\hline $3 \mathrm{H}-4,86-92$ & 23.66 & 4 & 0 & 42 & i & 0 & 0 & 0 & 0 & 1 & 6 \\
\hline $3 \mathrm{H}-5,79-85$ & 25.09 & 1 & 0 & 0 & 0 & 0 & 0 & 0 & 0 & 0 & 0 \\
\hline $3 \mathrm{H}-6,83-89$ & 26.63 & 0 & 0 & 2 & 4 & 0 & 0 & 0 & 0 & i & 12 \\
\hline $3 \mathrm{H}-\mathrm{CC}$ & 27.5 & 5 & 0 & 26 & 23 & 0 & 0 & 0 & 0 & 0 & 39 \\
\hline $4 \mathrm{H}-1.80-86$ & 28.1 & 10 & 0 & 13 & 0 & 0 & 0 & 1 & 0 & 5 & 231 \\
\hline $4 \mathrm{H}-2,80-86$ & 29.6 & 94 & 0 & 0 & 0 & 0 & 0 & 0 & 0 & 0 & 29 \\
\hline $4 \mathrm{H}-3.84-89$ & 31.14 & 107 & 1 & 32 & 8 & 0 & 0 & 0 & 0 & 0 & 20 \\
\hline $4 \mathrm{H}-4,71-83$ & 32.56 & 0 & 0 & 0 & 0 & 0 & 0 & 0 & 0 & 0 & 0 \\
\hline $4 \mathrm{H}-5,80-86$ & 34.1 & 17 & $i$ & 20 & 0 & 0 & 0 & 2 & 0 & 6 & 141 \\
\hline $4 \mathrm{H}-\mathrm{CC}$ & 36 & 37 & i & 9 & 4 & 0 & 0 & 0 & 0 & 0 & 149 \\
\hline $5 \mathrm{H}-1.78-84$ & 37.48 & 166 & 0 & 7 & 0 & 0 & 0 & 0 & 0 & 0 & 31 \\
\hline $5 \mathrm{H}-2,105-113$ & 39.27 & 162 & i & 18 & 10 & 0 & 0 & i & i & 1 & 5 \\
\hline $5 \mathrm{H}-3,106-113$ & 40.76 & 130 & i & 47 & I & 0 & 0 & 0 & 0 & i & 15 \\
\hline $5 \mathrm{H}-4,108-114$ & 42.28 & 112 & i & 24 & 5 & 0 & 0 & 0 & 0 & 0 & 16 \\
\hline $5 H-5,78-84$ & 43.48 & 306 & 0 & 8 & 1 & 0 & 0 & 0 & 0 & 0 & 10 \\
\hline $5 \mathrm{H}-6.77-83$ & 44.97 & 0 & 0 & 214 & 71 & 0 & 0 & 0 & 0 & 0 & 8 \\
\hline $5 \mathrm{H}-7,77-83$ & 46.47 & 97 & 4 & 88 & 52 & 0 & 0 & 0 & 0 & 0 & 21 \\
\hline SH-CC & 47.2 & 23 & 4 & 54 & 222 & 0 & 0 & 0 & 0 & 0 & 20 \\
\hline $6 \mathrm{H}-2,98-104$ & 48.68 & 124 & $i$ & 0 & 0 & 0 & 0 & 0 & 0 & 0 & 15 \\
\hline $6 \mathrm{H}-3,105-111$ & 50.25 & 3 & 0 & 0 & 0 & 0 & 0 & 0 & 0 & 0 & 27 \\
\hline $6 \mathrm{H}-4,9-14$ & 50.79 & 56 & 0 & 15 & $i$ & 0 & 0 & 0 & 0 & 3 & 165 \\
\hline $6 \mathrm{H}-5,10-15$ & 52.3 & 6 & 0 & 0 & 0 & 0 & 0 & 0 & 0 & 0 & 4 \\
\hline $6 \mathrm{H}-\mathrm{CC}$ & 56.06 & 45 & 3 & 6 & 0 & 0 & 0 & 0 & 0 & 0 & 7 \\
\hline $7 \mathrm{H}-1,83-87$ & 56.53 & 40 & i & 27 & i & 0 & 0 & 0 & 0 & 0 & 12 \\
\hline $7 \mathrm{H}-2,41-46$ & 58 & 53 & 3 & 22 & 2 & 0 & 0 & 3 & 0 & 0 & 56 \\
\hline $7 \mathrm{H}-3,66-70$ & 59 & 0 & 0 & 1 & 0 & 0 & 0 & 0 & 0 & 0 & 0 \\
\hline $7 \mathrm{H}-4,68-73$ & 60.52 & 119 & 4 & 18 & 0 & 0 & 0 & 0 & 0 & 0 & 22 \\
\hline $7 \mathrm{H}-5,43-48$ & 61.77 & 22 & 0 & 4 & 0 & 0 & 0 & 0 & 0 & 0 & 32 \\
\hline $7 \mathrm{H}-6,58-64$ & 63.42 & 50 & 0 & 0 & 0 & 0 & 0 & 0 & 0 & 0 & 7 \\
\hline $7 \mathrm{H}-8,65-70$ & 66.49 & 83 & 0 & 4 & 0 & 0 & 0 & 0 & 0 & 0 & 32 \\
\hline $7 \mathrm{H}-\mathrm{CC}$ & 66.52 & 39 & 0 & 2 & 0 & 0 & 0 & 0 & 0 & 0 & 29 \\
\hline $8 \mathrm{H}-2,108-112$ & 66.68 & 42 & 0 & 5 & 1 & 0 & 0 & 0 & 0 & 0 & 63 \\
\hline $8 \mathrm{H}-3,108-114$ & 68.18 & 40 & 0 & 2 & 9 & 0 & 0 & 0 & 0 & 0 & 45 \\
\hline $8 \mathrm{H}-4,45-51$ & 69.68 & 55 & 5 & 10 & 0 & 0 & 0 & 0 & 0 & 2 & 66 \\
\hline $8 \mathrm{H}-5,108-114$ & 71.18 & 52 & 0 & 23 & 2 & 1 & 0 & 0 & 0 & 2 & 54 \\
\hline $8 \mathrm{H}-6,108-116$ & 72.68 & 49 & 0 & 7 & 27 & 0 & 0 & 2 & 0 & 3 & 67 \\
\hline $8 \mathrm{H}-7.108-114$ & 74.18 & 25 & 0 & i & 0 & 0 & 0 & 0 & 0 & 11 & 290 \\
\hline $8 \mathrm{H}-8,69-76$ & 75.29 & 4 & 0 & 22 & 14 & 0 & 0 & 11 & 3 & 2 & 197 \\
\hline $8 \mathrm{H}-\mathrm{CC}$ & 75.4 & 41 & 0 & 12 & 3 & 0 & 0 & 0 & 0 & 0 & 15 \\
\hline $9 \mathrm{H}-1,80-86$ & 75.6 & 160 & 46 & 14 & 0 & 0 & 0 & 0 & i & 0 & 20 \\
\hline $9 \mathrm{H}-2,80-86$ & 77.1 & 6 & 0 & 38 & 0 & 0 & 0 & 0 & 0 & 3 & 48 \\
\hline $9 \mathrm{H}-3,80-86$ & 78.6 & 16 & 4 & 13 & 2 & 0 & 0 & 1 & 0 & 0 & 90 \\
\hline $9 \mathrm{H}-4,79-85$ & 80.09 & 48 & 3 & 24 & 6 & 0 & 0 & 0 & 0 & 3 & 42 \\
\hline $9 \mathrm{H}-5,87-93$ & 81.67 & 31 & 0 & 43 & 16 & 0 & I & 19 & 0 & 0 & 67 \\
\hline $9 \mathrm{H}-6,80-86$ & 83.1 & 73 & 0 & 29 & 9 & 0 & 0 & 3 & 0 & 0 & 56 \\
\hline $9 \mathrm{H}-7,80-86$ & 84.6 & 0 & 0 & 0 & 0 & 0 & 0 & 0 & 0 & 0 & 1 \\
\hline $9 \mathrm{H}-\mathrm{CC}$ & 84.65 & 185 & 112 & 0 & 0 & 0 & 0 & 0 & 0 & 0 & 0 \\
\hline $10 \mathrm{H}-1,47-58$ & 84.87 & 20 & 0 & 15 & 25 & 0 & 4 & i & 0 & 6 & 22 \\
\hline $10 \mathrm{H}-2,90-95$ & 85.92 & 0 & 0 & 0 & 0 & 0 & 0 & 0 & 0 & 0 & 0 \\
\hline $10 \mathrm{H}-3.90-95$ & 87.42 & i & 0 & 0 & 0 & 0 & 0 & 0 & 0 & 0 & 16 \\
\hline
\end{tabular}

3. gyre-margin fauna distinguished by high numbers of $G$. bulloides and $G$. eggeri (i.e., $N$. dutertrei) along with dextrally coiling specimens of $N$. pachyderma;

4. subtropical fauna dominated by Globigerinita glutinata or Globigerinoides ruber;

5. dissolution-susceptible tropical fauna marked by G. ruber and G. glutinata;

6. dissolution-resistant tropical fauna dominated by Globorotalia menardii and Pulleniatina obliquiloculata.

Planktonic foraminifers were recovered in all except three of the 122 samples studied from Holes 798A and 798B (Table 1 and Fig. 2). No samples were studied from Hole $798 \mathrm{C}$. About $19 \%$ of the samples analyzed are significantly dissolved (i.e., yielded fewer than 50 identifiable specimens), and hence were not used for environmental interpretation.

The majority of the planktonic foraminifers analyzed from Pleistocene sediments at Site 798 can be referred to the polar-subpolar assemblage of Thompson (1981) and are characterized by abundant specimens of Neogloboquadrina pachyderma, Globigerina bulloides, and Globigerina quinqueloba (Fig. 2 and Table 2). However, a significant portion of the samples from lower Pleistocene sediments contains large numbers of Globorotalia inflata, dextrally coiling $N$. pachyderma, and minor amounts $(<5 \%)$ of Globigerinoides spp. and Globorotalia menardii and clearly represent relatively warm transitional and gyre-margin assemblages based on Thompson's (1981) classification.

The Pleistocene planktonic foraminiferal assemblages of Site 798 can be further divided into several assemblage types and subtypes as summarized in Table 2. Type A assemblages are dominated by $50 \%$ or more sinistrally coiling specimens of Neogloboquadrina pachyderma and constitute approximately $43 \%$ of all the samples examined. These assemblages are indicative of polar-subpolar waters, where according to Bé and Thoderlund (1971) sinistral $N$. pachyderma thrives in water temperatures between $0^{\circ}$ and $9^{\circ} \mathrm{C}$. Type B assemblages include $50 \%$ or more Globigerina bulloides and represent 
Table 1 (continued).

\begin{tabular}{|c|c|c|c|c|c|}
\hline $\begin{array}{l}\text { Core, section, } \\
\text { interval (cm) }\end{array}$ & $\begin{array}{l}\text { Neogloboquadrina } \\
\text { pachyderma } \\
\text { sinistral }\end{array}$ & $\begin{array}{l}\text { Pulleniatina } \\
\text { sp. }\end{array}$ & Other & $\begin{array}{c}\text { Total } \\
\text { number }\end{array}$ & $\begin{array}{c}\text { Sample } \\
\text { type }\end{array}$ \\
\hline \multicolumn{6}{|l|}{ 128-798A. } \\
\hline $1 \mathrm{H}-1,108-114$ & 255 & 0 & 2 & 324 & $\mathrm{~A} 2$ \\
\hline $1 \mathrm{H}-2,77-83$ & 226 & 0 & 1 & 329 & A2 \\
\hline IH-3, 80-86 & 233 & 0 & 8 & 322 & A3 \\
\hline $1 \mathrm{H}-4,106-112$ & 266 & 0 & 0 & 351 & A3 \\
\hline IH- $5,84-90$ & 234 & 0 & 2 & 300 & A2 \\
\hline $\mathrm{IH}-\mathrm{CC}$ & 217 & 0 & 3 & 302 & A2 \\
\hline $2 \mathrm{H} \cdot 1,79-85$ & 2 & 0 & 0 & 301 & c \\
\hline $2 \mathrm{H}-2,79-85$ & 112 & 0 & 5 & 284 & B2 \\
\hline $2 \mathrm{H}-3,79-84$ & 10 & 0 & 0 & 34 & NA \\
\hline $2 \mathrm{H} \cdot \mathrm{CC}$ & 124 & 0 & 0 & 300 & B2 \\
\hline $3 \mathrm{H}-1.80-86$ & 221 & 0 & 1 & 241 & Al \\
\hline $3 \mathrm{H}-2,105-111$ & 18 & 0 & 0 & 19 & NA \\
\hline $3 \mathrm{H}-3,80-86$ & 316 & 0 & 0 & 321 & $\mathrm{Al}$ \\
\hline $3 \mathrm{H}-4,86-92$ & 193 & 0 & 3 & 250 & A3 \\
\hline $3 \mathrm{H}-5.79-85$ & 9 & 0 & 0 & 10 & NA \\
\hline $3 \mathrm{H}-6.83-89$ & 308 & 0 & 0 & 327 & Al \\
\hline $3 \mathrm{H}-\mathrm{CC}$ & 201 & 0 & 0 & 294 & A3 \\
\hline $4 \mathrm{H}-1,80-86$ & 23 & 0 & 0 & 283 & C \\
\hline $4 \mathrm{H}-2,80-86$ & 203 & 0 & 0 & 326 & B2 \\
\hline $4 \mathrm{H}-3,84-89$ & 128 & 0 & 6 & 303 & B2 \\
\hline $4 \mathrm{H}-4,71-83$ & 0 & 0 & 0 & 0 & NA \\
\hline $4 H-5,80-86$ & 44 & 0 & 2 & 233 & C \\
\hline $4 \mathrm{H}-\mathrm{CC}$ & 83 & 0 & 0 & 292 & c \\
\hline $5 \mathrm{H}-1,78-84$ & 58 & 0 & 0 & 262 & B1 \\
\hline $5 \mathrm{H}-2,105-113$ & 35 & 0 & 0 & 234 & BI \\
\hline $5 \mathrm{H}-3,106-113$ & 131 & 0 & i & 327 & B2 \\
\hline $5 \mathrm{H}-4,108-114$ & 158 & 0 & 4 & 320 & B2 \\
\hline $5 \mathrm{H}-5,78-84$ & 14 & 0 & i & 340 & BI \\
\hline $5 \mathrm{H}-6,77-83$ & 13 & 0 & 0 & 306 & EI \\
\hline $5 \mathrm{H}-7,77-83$ & 35 & 0 & 0 & 297 & El \\
\hline $5 \mathrm{H}-\mathrm{CC}$ & 23 & 0 & 0 & 346 & E2 \\
\hline $6 \mathrm{H} \cdot 2,98-104$ & 180 & 0 & 0 & 320 & B2 \\
\hline $6 \mathrm{H}-3,105-111$ & 68 & 0 & 0 & 98 & A4 \\
\hline $6 \mathrm{H}-4,9-14$ & 60 & 0 & 0 & 300 & C \\
\hline $6 \mathrm{H}-5,10-15$ & 78 & 0 & 0 & 88 & Al \\
\hline $6 \mathrm{H}-\mathrm{CC}$ & 251 & 0 & 1 & 313 & A2 \\
\hline $7 \mathrm{H}-1,83-87$ & 223 & 0 & 0 & 304 & $\mathrm{~A} 2$ \\
\hline $7 \mathrm{H}-2,41-46$ & 154 & 0 & 0 & 293 & A2 \\
\hline $7 \mathrm{H}-3,66-70$ & 5 & 0 & 0 & 6 & NA \\
\hline $7 \mathrm{H}-4,68-73$ & 147 & 0 & 0 & 310 & B2 \\
\hline $7 \mathrm{H}-5,43-48$ & 223 & 0 & 0 & 281 & A4 \\
\hline $7 \mathrm{H}-6,58-64$ & 215 & 0 & 0 & 272 & A2 \\
\hline $7 \mathrm{H}-8,65-70$ & 188 & 0 & 0 & 307 & A2 \\
\hline $7 \mathrm{H}-\mathrm{CC}$ & 258 & 0 & 2 & 330 & $\mathrm{~A} 2$ \\
\hline $8 \mathrm{H}-2,108-112$ & 185 & 0 & 0 & 296 & A4 \\
\hline $8 \mathrm{H}-3,108-114$ & 214 & 0 & i & 311 & A4 \\
\hline $8 \mathrm{H}-4,45-51$ & 157 & 0 & 0 & 295 & A4 \\
\hline $8 \mathrm{H}-5,108-114$ & 165 & 0 & 3 & 302 & A4 \\
\hline $8 \mathrm{H}-6,108-116$ & 211 & 0 & 2 & 368 & A4 \\
\hline $8 \mathrm{H}-7,108-114$ & 22 & 0 & 0 & 349 & C \\
\hline $8 \mathrm{H}-8,69-76$ & 26 & 0 & 9 & 288 & C \\
\hline $8 \mathrm{H}-\mathrm{CC}$ & 234 & 0 & 0 & 305 & A2 \\
\hline $9 \mathrm{H}-1,80-86$ & 65 & 0 & 4 & 310 & B1 \\
\hline $9 \mathrm{H}-2,80-86$ & 243 & 0 & 13 & 351 & A4 \\
\hline $9 \mathrm{H}-3,80-86$ & 176 & 0 & 1 & 303 & A4 \\
\hline $9 \mathrm{H}-4,79-85$ & 197 & 0 & 4 & 327 & A2 \\
\hline $9 \mathrm{H}-5,87-93$ & 150 & 0 & 0 & 327 & A4 \\
\hline $9 \mathrm{H}-6,80-86$ & 161 & 0 & 9 & 340 & A2 \\
\hline $9 \mathrm{H}-7,80-86$ & 14 & 0 & 0 & 15 & NA \\
\hline 9H-CC & 14 & 0 & 3 & 314 & BI \\
\hline $10 \mathrm{H}-1,47-58$ & 216 & 0 & 4 & 313 & $\mathrm{~A}^{3}$ \\
\hline $10 \mathrm{H}-2,90-95$ & 0 & 0 & 0 & 0 & NA \\
\hline $10 \mathrm{H}-3,90-95$ & 305 & 0 & 0 & 322 & Al \\
\hline
\end{tabular}

about $19 \%$ of the Site 798 samples. The type B assemblages can best be compared to the gyre-margin fauna of Thompson (1981), which he associated with the upwelling of Kuroshio waters around the subtropical divergence off Northeastern Honshu. Type C and D assemblages both represent transitional assemblages associated with the Tsushima Current water and together represent about $16.5 \%$ of the assemblages studied. By definition, type $\mathrm{C}$ assemblages include at least $15 \%$ dextrally coiling $N$. pachyderma, which is known to have optimum occurrence at temperatures between $10^{\circ}$ and $18^{\circ} \mathrm{C}$ (Bé and Thoderlund, 1971). Type D assemblages are similar to type C assemblages, but also include $20 \%$ or more Globorotalia inflata. The type D assemblages include sporadic occurrences $(<5 \%)$ of Globigerinoides spp., Globorotalia menardii, and Pulleniatina sp., representing transitional to tropical species that have previously been associated with the warm Tsushima Current (Bradshaw, 1959; Chinzei et al., 1987). Type E assemblages characterize about $2.5 \%$ of the samples studied and include more than 50\% Globigerina quinqueloba or Globigerinita spp. The paleoenvironmental significance of type $\mathrm{E}$ assemblages is not clear. However, because the dominant species are delicate forms that dissolve before other species such as G. bulloides and N. pachyderma, type $\mathrm{E}$ assemblages represent the best preserved samples recovered at Site 798 (Sautter and Thunell, 1989, and references therein).

\section{SEA-SURFACE TEMPERATURE AND CIRCULATION}

Planktonic foraminifers can be utilized to interpret past sea-surface temperatures through analysis of the variation in abundances of distinctive assemblage types (Table 2) and through characterization of the occurrence of selected species. In mid- to high-latitude regions the coiling ratio changes within the Neogloboquadrina pachyderma group are a well-established and powerful tool for the identification of latitudinal shifts of surface isotherms. $N$. pachyderma was first recognized as a paleoclimatic indicator by Ericson (1959) and Bandy $(1959,1960)$. Some authors consider the sinistrally and dextrally coiling forms of $N$. pachyderma to constitute two different species that thrive in cold polar-subpolar and warmer subtropical-transitional water masses, respectively. For example, Cifelli (1973) referred to dextrally coiling forms as Globigerina incompta (Cifelli, 1961) and explained that it is larger and has thinner walls than "true" N. pachyderma. Olsson (1974) referred to the larger late Pliocene and early Pleistocene forms of this group as Globorotalia pseudopachyderma. Olsson explained that $G$. incompta can coil both ways and represent warmer sea-surface temperatures than the consistently left-coiling $N$. pachyderma in the late Pleistocene. Other authors (Parker and Berger, 1971; Kennett and Srinivasan, 1980; Arikawa, 1983) generally believed that the dextral forms of $N$. pachyderma are genetically related to the subtropical Neogloboquadrina dutertrei. Kennett and Srinivasan (1980) also found the surface ultrastructure of the dextral and sinistral forms to be identical, whereas Olsson (1976) described them to be significantly different. Presently, I refer to both forms as N. pachyderma and agree with Cifelli (1973) that from the standpoint of environmental interpretation it does not matter if the sinistral and dextral forms represent one or two species. At Site 798 both sinistral and dextral forms of N. pachyderma s.l. occur in high abundances and provide the most important evidence of variations in sea-surface temperature, surface-water masses, and circulation in the Pleistocene of the Japan Sea.

\section{Sea-Surface Temperature Fluctuations in the Japan Sea: Evidence from Site 798}

The continuous occurrence of Neogloboquadrina pachyderma in sediments at Site 798 provides the first opportunity to examine a relatively complete record of Pleistocene variations in sea-surface temperature fluctuations in the Japan Sea. Variations in coiling direction of $N$. pachyderma at Site 798 reveal a complex pattern of warm/cold sea-surface temperature in the Japan Sea throughout the Pleistocene, and have implications for glacial/interglacial patterns in the Northern Pacific Ocean as a whole. Tentative correlations of the warm/cold events at Site 798 with the North Pacific CD shifts of Lagoe and Thompson (1988) are illustrated in Figure 3. The early Pleistocene at Site 798 is marked by a period dominated by dextrally coiling specimens of $N$. pachyderma as well as abundant Globorotalia inflata. This interval (Cores 128-798B-21X through 128-798A$13 \mathrm{H}$ ) is thought to be correlative with the CD 11 shift of Lagoe and Thompson (1988), which represents relatively warm sea-surface temperatures. Evidence of the onset of mid-latitude glaciation at 1.2 Ma (Kent et al., 1971) is recognized in Core 128-798A-13H as expressed by fluctuating surface conditions based on coiling shifts of $N$. pachyderma and supported by a shipboard nannofossil datum (last-appearance datum of Helicosphaera sellii; Core 128-798A13H-4; Ingle, Suyehiro, von Breymann, et al., 1990). The Pacific CD 9-10 shifts correspond to the interval between the 1.2 Ma datum in Core $128-798 \mathrm{~A}-13 \mathrm{H}$ and the Brunhes/Matuyama boundary in the low- 
Table 1 (continued).

\begin{tabular}{|c|c|c|c|c|c|c|c|c|c|c|c|}
\hline $\begin{array}{l}\text { Core, section, } \\
\text { interval (cm) }\end{array}$ & $\begin{array}{l}\text { Depth } \\
\text { (mbsf) }\end{array}$ & $\begin{array}{l}\text { Globigerina } \\
\text { bulloides }\end{array}$ & $\begin{array}{c}\text { Globigerina } \\
\text { bulloides } \\
\text { umbilicata }\end{array}$ & $\begin{array}{l}\text { Globigerina } \\
\text { quinqueloba }\end{array}$ & $\begin{array}{l}\text { Globigerinita } \\
\text { spp. }\end{array}$ & $\begin{array}{l}\text { Globorotalia } \\
\text { inflata }\end{array}$ & $\begin{array}{l}\text { Globorotalia } \\
\text { menardii }\end{array}$ & $\begin{array}{c}\text { Globigerinoides } \\
\text { ruber }\end{array}$ & $\begin{array}{l}\text { Globigerinoides } \\
\text { sp. }\end{array}$ & $\begin{array}{l}\text { Neogloboquadrina } \\
\text { dutertrei }\end{array}$ & $\begin{array}{l}\text { Neogloboquadrina } \\
\text { pachyderma } \\
\text { dextral }\end{array}$ \\
\hline \multicolumn{12}{|l|}{ 128-798A- } \\
\hline $10 \mathrm{H}-4,84-90$ & 88.86 & 0 & 0 & II & 0 & 0 & 0 & 0 & 0 & 0 & 22 \\
\hline $10 \mathrm{H}-5,84-90$ & 90.36 & 0 & 0 & 0 & 0 & 0 & 0 & 0 & 0 & 0 & 0 \\
\hline 10H-6, 84-90 & 91.86 & 14 & 0 & 0 & 0 & 0 & 0 & 0 & 0 & 0 & 23 \\
\hline $10 \mathrm{H}-7.75-80$ & 93.27 & 0 & 0 & 9 & 0 & o & 0 & 0 & 0 & 7 & 85 \\
\hline $10 \mathrm{H}-8,75-80$ & 94.77 & 2 & 0 & 28 & 1 & 0 & 0 & 0 & 0 & 0 & 40 \\
\hline $10 \mathrm{H}-\mathrm{CC}$ & 94.85 & 1 & 0 & 33 & 1 & 0 & 0 & 0 & 0 & 0 & 36 \\
\hline $11 \mathrm{H}-1.89-95$ & 94.99 & 8 & 0 & 3 & 0 & 0 & 0 & 0 & 0 & 0 & 2 \\
\hline $11 \mathrm{H}-2,82-88$ & 96.42 & 0 & 0 & 0 & 0 & 0 & 0 & 0 & 0 & 0 & 0 \\
\hline IIH-3, 80-86 & 97.9 & 0 & 0 & 42 & 0 & 0 & 0 & 0 & 0 & 0 & 63 \\
\hline IIH-4, 78-84 & 99.38 & 46 & 1 & 17 & 0 & 0 & 0 & 0 & 0 & 0 & 60 \\
\hline IIH-5, 78-84 & 100.88 & 44 & 4 & 3 & 4 & 0 & 0 & 0 & 0 & 0 & 66 \\
\hline $11 \mathrm{H}-6.80-86$ & 102.4 & 67 & 5 & 36 & 1 & 0 & 0 & 0 & 0 & 0 & 53 \\
\hline $11 \mathrm{H}-7,79-85$ & 103.89 & 1 & 0 & 35 & 0 & 0 & 0 & 0 & 0 & 0 & 214 \\
\hline $11 \mathrm{H}-\mathrm{CC}$ & 104.5 & 8 & 2 & 16 & 0 & 0 & 0 & 0 & 0 & 0 & 92 \\
\hline $12 \mathrm{H}-2,79-85$ & 105.55 & 4 & 0 & 1 & 0 & 0 & 0 & 0 & 0 & 0 & 5 \\
\hline $12 \mathrm{H}-3,99-105$ & 107.06 & 0 & 0 & 0 & 0 & 0 & 0 & 0 & 0 & 0 & 1 \\
\hline $12 \mathrm{H}-4,103-109$ & 108.6 & 0 & 0 & 0 & 0 & 0 & 0 & 0 & 0 & 0 & 0 \\
\hline $12 \mathrm{H}-5.79-85$ & 109.86 & 0 & 0 & 1 & 0 & 0 & 0 & 0 & 0 & 0 & 0 \\
\hline $12 \mathrm{H}-6,96-101$ & 111.53 & 3 & i & 0 & 0 & 0 & 0 & 0 & 0 & 0 & 4 \\
\hline $12 \mathrm{H}-7,83-89$ & 112.9 & 64 & 0 & 0 & 0 & 0 & 0 & 0 & 0 & 0 & 37 \\
\hline $12 \mathrm{H}-8,82-89$ & 114.39 & 36 & i & 0 & 0 & 0 & 0 & 0 & 0 & 0 & 42 \\
\hline $12 \mathrm{H}-\mathrm{CC}$ & 114.42 & 0 & 0 & 0 & 0 & 0 & 0 & 0 & 0 & 0 & 0 \\
\hline $13 \mathrm{H}-1.110-116$ & 114.5 & 30 & 0 & 6 & 0 & 0 & 0 & 0 & 0 & 0 & 21 \\
\hline $13 \mathrm{H}-2,79-85$ & 115.69 & 43 & 0 & 0 & 0 & 0 & 0 & 0 & 0 & 0 & 8 \\
\hline $13 \mathrm{H}-3.80-86$ & 117.2 & 106 & 3 & 55 & 4 & 0 & 0 & 0 & 0 & 0 & 112 \\
\hline $13 \mathrm{H}-4,81-87$ & 118.71 & 32 & 0 & 2 & 0 & 0 & 0 & 0 & 0 & 0 & 43 \\
\hline $13 \mathrm{H}-5.85-91$ & 120.25 & 0 & 0 & 0 & 0 & 0 & 0 & 0 & 0 & 0 & 0 \\
\hline $13 \mathrm{H}-6,86-91$ & 121.75 & 0 & 0 & 0 & 0 & 0 & 0 & 0 & 0 & 0 & 2 \\
\hline $13 \mathrm{H} \cdot \mathrm{CC}$ & 122 & 45 & & 71 & 6 & 0 & 0 & 0 & 0 & 0 & 152 \\
\hline $14 \mathrm{H}-2,104-110$ & 124.55 & 28 & 0 & 9 & i & o & 0 & 0 & 0 & 1 & 229 \\
\hline $14 \mathrm{H}-3,123-129$ & 126.24 & 0 & 0 & 0 & 0 & 0 & 0 & 0 & 0 & 0 & 1 \\
\hline $14 \mathrm{H}-4,134-140$ & 127.85 & 78 & i & 43 & II & & 0 & 0 & 0 & 1 & 111 \\
\hline $14 \mathrm{H}-5,123-129$ & 129.24 & 0 & 0 & 88 & ii & 0 & 0 & 0 & 0 & 0 & 132 \\
\hline $14 \mathrm{H}-6,124-130$ & 130.75 & 19 & 0 & 32 & 0 & 162 & i & 6 & & 2 & 63 \\
\hline $14 \mathrm{H}-7,7-13$ & 131.08 & 8 & 0 & 8 & 0 & & 0 & 0 & 0 & 0 & 37 \\
\hline $14 \mathrm{H}-8,7-13$ & 132.58 & 16 & 0 & 13 & 0 & 0 & 0 & 1 & 0 & 0 & 222 \\
\hline $14 \mathrm{H}-\mathrm{CC}$ & 133 & 0 & 0 & 0 & i & 0 & 0 & 0 & 0 & 0 & 2 \\
\hline $15 \mathrm{H}-1,86-92$ & 133.66 & 12 & 0 & 7 & 0 & 0 & 0 & 0 & 0 & 0 & 47 \\
\hline $15 \mathrm{H}-2,86-92$ & 135 & 106 & 0 & 14 & 0 & 26 & 0 & 0 & 0 & 0 & 73 \\
\hline $15 \mathrm{H}-3,86-92$ & 136.56 & 8 & 0 & 0 & 0 & 0 & 0 & 0 & 0 & 0 & 6 \\
\hline $15 \mathrm{H}-4,86-92$ & 138.06 & 216 & 2 & 21 & 0 & 0 & 0 & 0 & 0 & 0 & 46 \\
\hline $15 \mathrm{H}-5,68-74$ & 139.38 & 185 & 0 & 0 & 0 & 0 & 0 & 0 & 0 & 1 & 87 \\
\hline $15 \mathrm{H}-6,80-86$ & 141 & 59 & 0 & 10 & 0 & 165 & 0 & 0 & 0 & 0 & 50 \\
\hline $15 \mathrm{H}-7.80-86$ & 142.5 & 75 & 0 & 7 & 1 & 122 & 0 & 1 & 0 & 0 & 70 \\
\hline $15 \mathrm{H}-8,80-86$ & 144 & 0 & 0 & 0 & 0 & 7 & 0 & 0 & 0 & 0 & 0 \\
\hline $15 \mathrm{H}-\mathrm{CC}$ & 146 & 21 & 0 & 2 & 0 & 0 & 0 & 0 & 0 & 0 & 13 \\
\hline \multicolumn{12}{|l|}{ 128-798B- } \\
\hline $16 X-5,5-11$ & 147.83 & 203 & 0 & 0 & 0 & 0 & 0 & 0 & 0 & 0 & 80 \\
\hline $16 \mathrm{X}-\mathrm{CC}$ & 150 & 61 & 0 & 39 & 0 & 51 & 0 & 0 & 0 & 0 & 88 \\
\hline $17 X-2,80-86$ & 153.88 & 25 & 0 & 4 & 0 & 0 & 0 & 0 & 0 & 0 & 0 \\
\hline $17 \mathrm{X}-\mathrm{CC}$ & 160 & 9 & 0 & 0 & 0 & 0 & 0 & 0 & 0 & 0 & 2 \\
\hline $18 X-2,79-85$ & 164.2 & 26 & 0 & 0 & 0 & 0 & 0 & 0 & 0 & 1 & 21 \\
\hline $18 \mathrm{X}-\mathrm{CC}$ & 170 & 175 & 1 & 10 & 0 & 0 & 0 & 0 & 0 & 0 & 86 \\
\hline $19 X-4,79-85$ & 176.89 & 3 & 0 & 0 & 0 & 0 & 0 & 0 & 0 & 0 & 0 \\
\hline 19X-CC & 180 & 172 & 7 & 19 & 2 & 0 & 0 & 0 & 0 & 0 & 97 \\
\hline $20 X-5,80-86$ & 187.57 & 3 & 0 & 0 & 0 & 0 & 0 & 0 & 0 & 0 & 0 \\
\hline $20 \mathrm{X}-\mathrm{CC}$ & 192.86 & 176 & 19 & 28 & 0 & 0 & 0 & 0 & 0 & 0 & 59 \\
\hline $21 \mathrm{X}-\mathrm{CC}$ & 200 & 38 & 0 & 10 & 1 & 0 & 0 & 0 & 0 & 0 & 8 \\
\hline $22 \mathrm{X}-\mathrm{CC}$ & 210 & 25 & 0 & 1 & 0 & 0 & 0 & 0 & 0 & 0 & 7 \\
\hline
\end{tabular}

Note: "Other" includes deformed and unidentifiable species. Sample type: $\mathrm{A} 1-\mathrm{A} 4=$ polar-subpolar: $\mathrm{B} 1-\mathrm{B} 3=$ gyre-margin: $\mathrm{C}$ and $\mathrm{D}=$ transitional: $\mathrm{E} 1-\mathrm{E} 2=$ ? well preserved: $\mathrm{NA}=$ not assigned due to bad preservation.

ermost portion of Core 128-798A-9H. In this interval the coiling dominance shifts attest to fluctuating surface conditions. The record of sea-surface temperature changes in the Brunhes Magnetochron at Site 798 includes numerous cold/warm events. These events in the Japan Sea are clearly more complex than the patterns of CD 1-8 shifts present in the generalized North Pacific curve of Lagoe and Thompson (1988). The coiling shift patterns at Site 798 greatly resemble the climatic fluctuations reported by Kent et al. (1971) and Olsson (1974), and can best be compared to the Brunhes record of glaciation/deglaciation and ice volume changes in the Northern Hemisphere.

Figure 4 illustrates the similarity of the interpreted Brunhes sea-surface temperature changes at Site 798 to the global glaciation/deglaciation curve of Imbrie et al. (1984) and Prell et al. (1986) for the Northern Hemisphere based on studies of oxygen isotopes and implied fluctuations in volumes of polar ice. Approximate positions of various oxygen isotopic stage boundaries are estimated in Table 3 and Figure 4 based on one to one comparison of cold/warm stages with coiling dominance shifts of Neogloboquadrina pachyderma. At Site 798 the estimated correlation of oxygen isotope stages and Japan Sea sea-surface temperatures suggests that oxygen isotope stage $1(0-12 \mathrm{Ka})$ is completely missing in Hole 798A, but is apparently present in Hole 798C (Dunbar et al., this volume). Stages 2 (12-24 Ka) and 3 (24-59 Ka) are probably present; however, the stage $2 / 3$ boundary can not be identified with certainty. Late Brunhes stages 4-11 (59-423 Ka) appear to be well represented in the Japan Sea in terms of the estimated sea-surface temperature record at Site 798. Deglaciation stages 5, 7, 9, and 11 appear to be expressed as events marked by the dominance of dextral $N$. pachyderma, in turn implying strong influence of the Tsushima Current at times of high sea-level stand and complete faunal turnover. The boundaries of the early Brunhes isotope stages 12-19 (423-736 Ka) are estimated with less confidence because of the less definitive $N$. pachyderma coiling patterns during this time. Stage $17(659-689 \mathrm{Ka})$ is an exception within this latter interval, and it can be readily separated from the other stages as the most prominent warm event in the early Brunhes Magnetochron. During the period represented by stages $12-19$, the Tsushima Current apparently entered the Japan Sea, but was not strong enough to signifi- 
Table 1 (continued).

\begin{tabular}{|c|c|c|c|c|c|}
\hline $\begin{array}{l}\text { Core, section, } \\
\text { interval }(\mathrm{cm})\end{array}$ & $\begin{array}{l}\text { Neogloboquadrina } \\
\text { pachyderma } \\
\text { sinistral }\end{array}$ & $\begin{array}{l}\text { Pulleniatina } \\
\text { sp. }\end{array}$ & Other & $\begin{array}{c}\text { Total } \\
\text { number }\end{array}$ & $\begin{array}{c}\text { Sample } \\
\text { type }\end{array}$ \\
\hline \multicolumn{6}{|l|}{ 128-798A. } \\
\hline $10 \mathrm{H}-4.84-90$ & 265 & 0 & 2 & 300 & Al \\
\hline $10 \mathrm{H}-5,84-90$ & 1 & 0 & 0 & 1 & NA \\
\hline $10 \mathrm{H}-6,84-90$ & 256 & 0 & 0 & 293 & Al \\
\hline $10 \mathrm{H}-7,75-80$ & 234 & 0 & 1 & 336 & A4 \\
\hline $10 \mathrm{H}-8,75-80$ & 267 & 0 & 1 & 339 & A4 \\
\hline $10 \mathrm{H}-\mathrm{CC}$ & 236 & 0 & 1 & 308 & A4 \\
\hline $11 \mathrm{H}-1.89-95$ & 183 & 0 & i & 197 & Al \\
\hline $11 \mathrm{H}-2,82-88$ & 0 & 0 & 0 & 0 & NA \\
\hline $11 \mathrm{H}-3,80-86$ & 222 & 0 & 2 & 329 & A4 \\
\hline IIH-4. 78-84 & 242 & 0 & 0 & 366 & A4 \\
\hline $11 \mathrm{H}-5,78-84$ & 269 & 0 & 1 & 391 & A4 \\
\hline $11 \mathrm{H}-6,80-86$ & 138 & 0 & II & 311 & A4 \\
\hline $11 \mathrm{H}-7.79-85$ & 118 & 0 & 0 & 368 & c \\
\hline 11H-CC & 192 & 0 & 0 & 311 & A4 \\
\hline $12 \mathrm{H}-2,79-85$ & 277 & 0 & 0 & 287 & Al \\
\hline $12 \mathrm{H}-3,99-105$ & 4 & 0 & 0 & 5 & NA \\
\hline $12 \mathrm{H}-4,103-109$ & 1 & 0 & 0 & 1 & NA \\
\hline $12 \mathrm{H}-5,79-85$ & 8 & 0 & 0 & 9 & NA \\
\hline $12 \mathrm{H}-6,96-101$ & 202 & $\mathbf{0}$ & 0 & 210 & Al \\
\hline $12 \mathrm{H}-7.83-89$ & 113 & 0 & 0 & 214 & $\mathrm{~A} 2 / \mathrm{B} 2$ \\
\hline $12 \mathrm{H}-8,82-89$ & 117 & 0 & 0 & 196 & A4 \\
\hline $12 \mathrm{H}-\mathrm{CC}$ & 2 & 0 & 0 & 2 & NA \\
\hline $13 \mathrm{H}-1,110-116$ & 246 & 0 & 1 & 304 & Al \\
\hline $13 \mathrm{H}-2,79-85$ & 7 & 0 & 0 & 58 & B1 \\
\hline $13 \mathrm{H}-3,80-86$ & 28 & $\mathbf{0}$ & 0 & 308 & B3 \\
\hline $13 \mathrm{H}-4,81-87$ & 236 & 0 & 0 & 313 & A4 \\
\hline $13 \mathrm{H}-5,85-91$ & 1 & 0 & 0 & I & NA \\
\hline $13 \mathrm{H}-6,86-91$ & 0 & 0 & 0 & 2 & NA \\
\hline $13 \mathrm{H}-\mathrm{CC}$ & 33 & 0 & 0 & 316 & c \\
\hline $14 \mathrm{H}-2,104-110$ & 14 & 0 & 2 & 284 & C \\
\hline $14 \mathrm{H}-3,123-129$ & 0 & 0 & 0 & 1 & NA \\
\hline $14 \mathrm{H}-4,134-140$ & 45 & 0 & 7 & 345 & D \\
\hline $14 \mathrm{H}-5,123-129$ & 63 & 0 & 11 & 305 & C \\
\hline $14 \mathrm{H}-6,124-130$ & 0 & 5 & 6 & 296 & D \\
\hline $14 \mathrm{H}-7,7-13$ & 2 & 0 & 1 & 179 & D \\
\hline $14 \mathrm{H}-8,7-13$ & 13 & 0 & 1 & 266 & c \\
\hline $14 \mathrm{H}-\mathrm{CC}$ & 1 & 0 & 0 & 5 & NA \\
\hline $15 \mathrm{H}-1,86-92$ & 12 & 0 & 0 & 78 & C \\
\hline $15 \mathrm{H}-2,86-92$ & 9 & 0 & 1 & 229 & D \\
\hline $15 \mathrm{H}-3,86-92$ & 4 & 1 & 0 & 19 & $\mathrm{NA}$ \\
\hline $15 \mathrm{H}-4,86-92$ & 9 & 0 & 2 & 296 & $\mathrm{~B} 1 / \mathrm{C}$ \\
\hline $15 \mathrm{H}-5.68-74$ & 5 & 0 & 1 & 279 & $\mathrm{~B} 3 / \mathrm{C}$ \\
\hline $15 \mathrm{H}-6,80-86$ & 2 & 0 & 2 & 288 & D \\
\hline $15 \mathrm{H}-7,80-86$ & 6 & 0 & 0 & 282 & D \\
\hline $15 \mathrm{H}-8,80-86$ & 0 & 0 & 0 & 7 & NA \\
\hline $15 \mathrm{H}-\mathrm{CC}$ & 37 & 0 & 0 & 74 & $\mathrm{~A} 2$ \\
\hline \multicolumn{6}{|l|}{ 128-798B- } \\
\hline $16 x-5,5-11$ & 2 & 0 & 0 & 285 & $\mathrm{~B} 1 / \mathrm{C}$ \\
\hline $16 \times-C C$ & 38 & 0 & 0 & 277 & D \\
\hline $17 X-2,80-86$ & 1 & 0 & 0 & 31 & NA \\
\hline $17 \times-C C$ & 1 & 0 & 0 & 12 & NA \\
\hline $18 X-2,79-85$ & 3 & 0 & 1 & 52 & $\mathrm{~B} 3 / \mathrm{C}$ \\
\hline $18 \mathrm{X}-\mathrm{CC}$ & 24 & 0 & 2 & 298 & $\mathrm{~B} 3 / \mathrm{C}$ \\
\hline $19 X-4,79-85$ & 0 & 0 & 0 & 3 & NA \\
\hline $19 \mathrm{X}-\mathrm{CC}$ & 18 & 0 & 0 & 315 & $\mathrm{~B} 3 / \mathrm{C}$ \\
\hline $20 \times-5,80-86$ & 0 & 0 & 0 & 3 & NA \\
\hline $20 \mathrm{X}-\mathrm{CC}$ & 16 & 0 & 0 & 298 & B $3 / \mathrm{C}$ \\
\hline $21 \mathrm{X}-\mathrm{CC}$ & 123 & 0 & 0 & 180 & A2 \\
\hline $22 \mathrm{X}-\mathrm{CC}$ & 9 & 0 & 0 & 42 & NA \\
\hline
\end{tabular}

cantly alter the faunal character of the surface waters as it did in late Brunhes time. In summary, the pattern of implied fluctuations of the Pleistocene sea-surface temperatures in the Japan Sea based on variations in planktonic foraminifers correlates well with the global pattern of Pleistocene climate change based on oxygen isotope analyses.

\section{DISCUSSION}

The species of planktonic foraminifers present in Quaternary sediments of the Japan Sea are extant in the modern Pacific Ocean and provide specific insight into the paleoceanography of the sea. Comparison of the Pleistocene foraminiferal assemblages at Site 798 to models developed for the seasonal succession of foraminifers in Holocene sediment-trap samples from the Northern Pacific Ocean allows important ecological interpretations of the fossil faunas. Holocene sediment-trap samples commonly reflect specimens collected during cold (winter/spring) or warm (summer/fall) seasons, in contrast to fossil fauna in sediment samples, which commonly represent homogenized assemblages representing hundreds or even thousands of years. Prolonged changes of climate (i.e., long-term shifts of latitudinal isotherms) affect the seasonality in the mid-latitude regions more than in the equatorial and polar regions. During an extended glacial event in the mid-latitude regions weaker seasonality can lead to production and preservation for many years of cold-water assemblages, which are dominated by cold-water fauna similar to winter/spring accumulations; whereas, strong seasonality during a prolonged deglacial event in the same region can potentially lead to many years of accumulation of more diverse assemblages of planktonic foraminifers with compositions similar to summer/fall seasonal assemblages in the modern ocean. Thus, the seasonal accumulation of Holocene foraminifers and assemblages in older deep-sea sediments may be compared for the purpose of environmental interpretation.

Reynolds and Thunell (1985) and Sautter and Thunell (1989) discussed a model to explain the seasonal succession of planktonic foraminifers found in sediment-trap samples collected at Northeast Pacific station $\mathrm{P}\left(50^{\circ} \mathrm{N}, 145^{\circ} \mathrm{W} ; 3800 \mathrm{~m}\right.$ water depth). Globigerina quinqueloba, Globigerinita glutinata, and Neogloboquadrina pachyderma are the most abundant species in these samples and have annual peak productions in both the spring and fall. Sinistrally coiling forms of $N$. pachyderma have their peak abundance in the spring, when the upper water column is isothermal, cold, and nutrient rich as a result of Ekman-induced upwelling within the Gulf of Alaska Gyre. Dextrally coiling forms of $N$. pachyderma become more dominant in the fall, when the water column is well stratified following warming of the surface waters in the summer months. Globigerina bulloides blooms in the late spring and early summer months, responding to the upwelling of nutrients in the spring and the gradual warming of the surface temperatures. Below station $\mathrm{P}$ on the seafloor, the surface sediments include $90 \%$ sinistral $N$. pachyderma and $10 \%$ G. bulloides, attesting to the severe dissolution of the planktonic foraminiferal assemblages prior to deposition (Sautter and Thunell, 1989). If planktonic foraminifers produced for several years could be deposited without significant dissolution, all the major species occurring in the surface waters would be present in variable abundances. Therefore, the older deep-sea samples that are dominated by sinistral $N$. pachyderma and low abundances of other species almost certainly represent assemblages that have been altered by dissolution and do not accurately reflect the nature of the water column at the time of their deposition. However, samples that include high abundances of $G$. bulloides, $G$. quinqueloba, G. glutinata, and dextral $N$. pachyderma may closely resemble the original assemblages and can be used with more certainty for interpretation of the surface environments.

Tables 1 and 2 and Figures 2 and 3 summarize the result of analysis of the planktonic foraminifers at Site 798. The type A assemblages, which represent cold $\left(<10^{\circ} \mathrm{C}\right)$ polar-subpolar surface waters (Thompson, 1981), characterize $43 \%$ of the Site 798 foraminiferal assemblages. Type A assemblages are dominated by sinistrally coiling specimens of Neogloboquadrina pachyderma and likely include the most dissolved assemblages recovered at Site 798. The type A assemblages are divided into subtypes (A1-A4) based on the presence of Globigerina bulloides, Globigerina quinqueloba, and dextrally coiling $N$. pachyderma as secondary dominant components (Table 2). The A1 assemblages, which constitute about $9.1 \%$ of all samples analyzed, are composed almost entirely of sinistral $N$. pachyderma and are therefore assumed to be strongly dissolved in comparison with the A2-A4 assemblages. About $19 \%$ of the studied samples included fewer than 50 specimens or were completely barren, and these were not assigned to any assemblage types (NA; Table 1). Therefore, it can be concluded that approximately $28 \%$ (including both A1 and NA types; Table 1) of the Site 798 samples have experienced severe dissolution, most likely caused by local shallowing of the $\mathrm{CCD}$ as a result of cooler temperatures in the water column.

Most of the Al and NA assemblages are scattered throughout the section, but they are most common between Cores 128-798A-10H and $128-798 \mathrm{~A}-14 \mathrm{H}$. However, one highly dissolved zone was noted in Core 128-798A-12H (Fig. 2) just above the 1.2-Ma datum thought 
Table 2. Assigned planktonic foraminiferal assemblage types for Site 798.

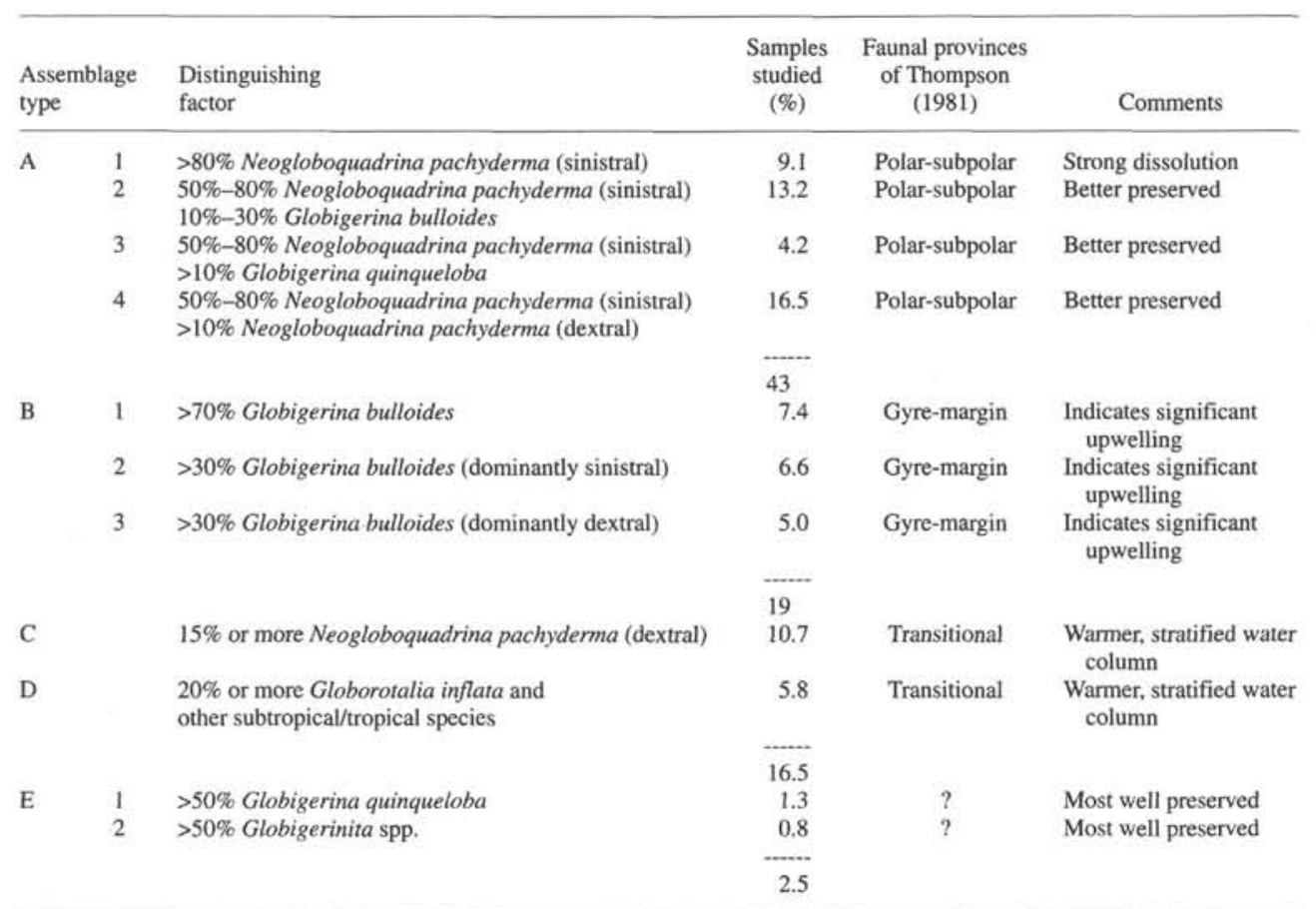

Note: Assemblage type assigned using the given distinguishing factors and individual evaluation of samples. The comments are based on interpretations of Sautter and Thunell (1989). The remaining 19\% of the samples are too dissolved or barren and are not assigned to any sample types.

to mark the onset of severe Northern Hemisphere glaciation (Kent et al., 1971). In general the type A assemblages are more abundant in the upper Pleistocene above Core 128-798A-9H which suggests restricted flow of the Tsushima Current into the Japan Sea following the onset of glaciation in the Northern Hemisphere.

The type B assemblages are dominated by Globigerina bulloides and may be divided into subtypes based on presence of lesser amounts of sinistrally and dextrally coiling Neogloboquadrina pachyderma (Table 2). These assemblages best resemble the gyre-margin assemblages of Thompson (1981). They characterize 19\% of the analyzed samples and indicate increased upwelling and possibly slightly warmer surface temperatures, because in the modern environments this species is found in high numbers in association with upwelling and increased surface temperature in the late spring and early summer (Reynolds and Thunell, 1985; Sautter and Thunell, 1989). Table 1 and Figure 2 illustrate that type B assemblages are especially abundant in two intervals at Site 798; one in the lower Pleistocene (Cores 128798A-15H through 128-798B-20X, 1.6-1.3 Ma; Fig. 3) and the other in the upper Pleistocene (Cores 128-798A-4H through 128-798A-6H, isotopic stages 6-11, 0.4-0.18 Ma; Fig. 4). The presence of type B assemblages suggests periods of warmer temperatures, higher sea levels, and increased interaction between the Tsushima and Liman currents similar to the activity along the subtropical gyre-margin and Oyashio convergence in the adjacent Pacific Ocean. The respective dominance of dextrally vs. sinistrally coiling $N$. pachyderma in the B2 and B3 subtypes attests to the mixed nature of the surface waters and interaction between warm- and cold-water masses at approximately $1.6-1.3$ and $0.4-0.18 \mathrm{Ma}$.

The type $\mathrm{C}$ and $\mathrm{D}$ assemblages are dominated by high numbers of dextrally coiling Neogloboquadrina pachyderma and characterize $16.5 \%$ of the samples examined at Site 798. Type D assemblages are distinguished by significant numbers of Globorotalia inflata and minor amounts of other subtropical-tropical species. Both type $\mathrm{C}$ and $\mathrm{D}$ assemblages are considered to be equivalent to Thompson's (1981) warm transitional assemblage and appear mainly in the lower Pleis- tocene below the 1.2-Ma datum, and presumably prior to the onset of glaciation in the Northern Hemisphere (Kent et al., 1971). In the modern Northern Pacific Ocean dextrally coiling forms of $N$. pachyderma bloom in the fall, when the surface waters are warmer and the water column is well stratified (Reynolds and Thunell, 1985; Sautter and Thunell, 1989). Similarly, transitional water masses are warmer at the surface and better stratified and, therefore, provide optimum conditions for blooms of dextral N. pachyderma. Hence, the C and D assemblages, like the type B assemblages, are interpreted as representing the increased flow of the warm Tsushima Current in the Japan Sea. However, they suggest the dominance of the Tsushima Current and almost complete faunal turnover. It can be concluded that the warm transitional waters of the Tsushima Current dominated the hydrography of the southern Japan Sea during the early Pleistocene but affected the sea only rarely in the late Pleistocene as lowered sea levels restricted communication with the open Pacific.

Type E assemblages occur in Core 128-798A-5H (oxygen isotope stage 9, $0.3 \mathrm{Ma}$; Fig. 4). They constitute about $2.5 \%$ of the samples studied and are dominated by both Globigerina quinqueloba and Globigerinita spp. G. quinqueloba dominates the E1 assemblages, whereas Globigerinita spp. are the main component of the E2 assemblages. These latter two species are delicate and dissolve more readily than robust forms such as Neogloboquadrina pachyderma (Berger, 1968; Thunell and Honjo, 1981). Thompson (1981) suggested that Globigerinita glutinata is abundant in subtropical assemblages, whereas G. quinqueloba occurs mostly in polar-subpolar waters. Therefore, it is possible that the type E assemblages appeared as a result of the mixed nature of the surface waters at approximately $0.3 \mathrm{Ma}$, as supported by presence of the type B assemblages between 0.4 and $0.18 \mathrm{Ma}$. Regardless of the nature of the surface conditions and because both G. quinqueloba and Globigerinita spp. are susceptible to dissolution, it is possible to conclude that at about $0.3 \mathrm{Ma}$ good conditions for the preservation of planktonic foraminifers existed in the Japan Sea. The upwelling suggested by the occurrence of the type B samples in Cores 128-798A-4H through 128-798A-6H (0.18-0.4 

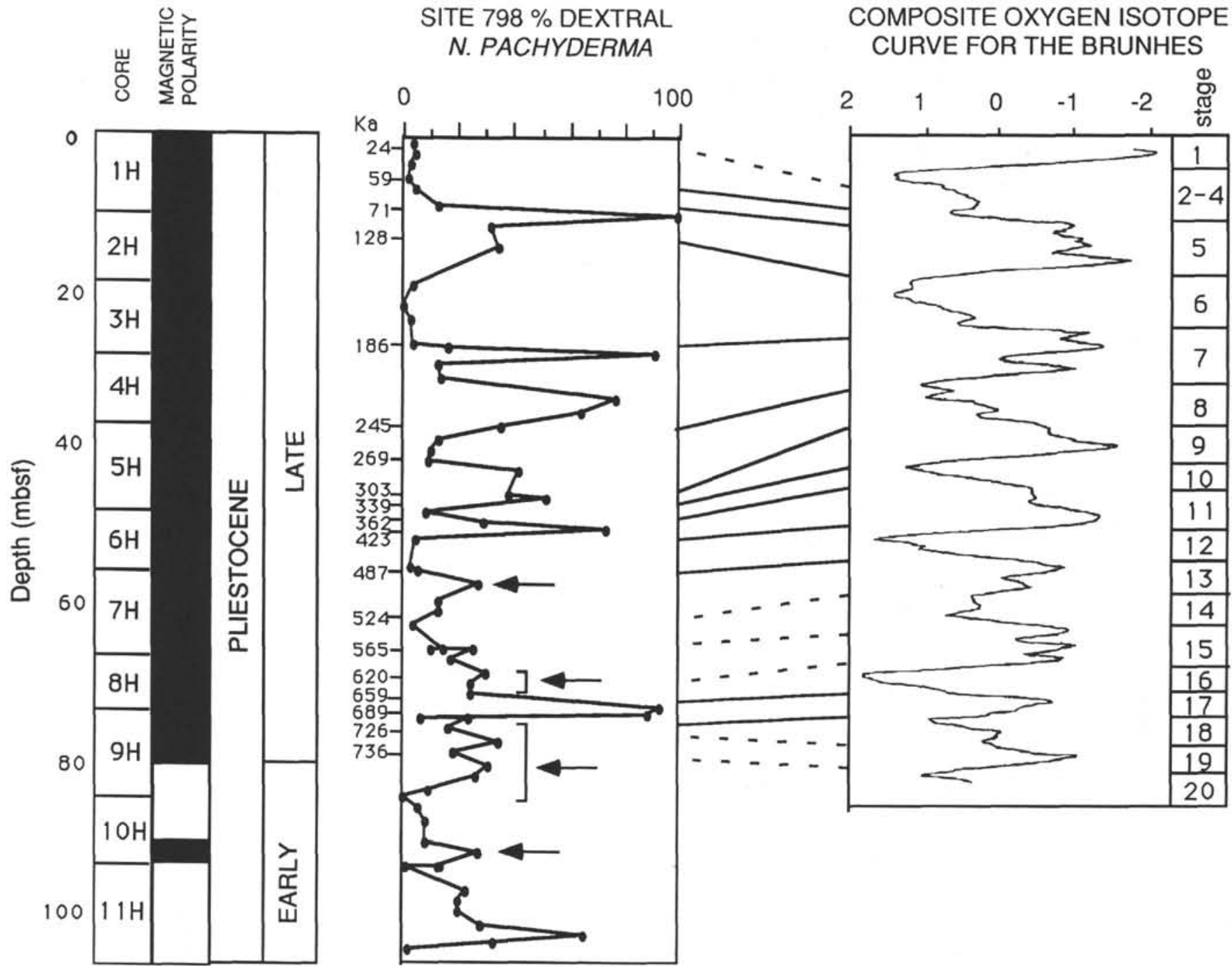

Figure 4. Correlation of variation in Neogloboquadrina pachyderma coiling dominance at Site 798 with oxygen isotope stages and age datums of Imbrie et al. (1984) and Prell et al. (1986). The dominance of dextrally coiling specimens of $N$. pachyderma is interpreted as evidence of warm sea-surface temperatures. Magnetostratigraphy from Ingle, Suyehiro, von Breymann, et al. (1990). The dashed lines show tentative correlations. Arrows point to samples that include tropical and/or transitional taxa indicating the presence of Tsushima Current waters.

Ma; Fig. 4) is coincident with the good-preservation conditions in Core 128-798 A-5H (0.3 Ma; Fig. 4) and indicates a higher production and flux rate for planktonic foraminifers at this time in the Japan Sea.

The changes in the pattern of coiling dominance of Neogloboquadrina pachyderma at Site 798 (Figs. 3 and 4) imply a major change in the hydrographic regime of the Japan Sea between the early and late early Pleistocene (1.2 Ma), as evidenced by the switch from the dominance of type B, C, and D assemblages to the common occurrence of type $A$ assemblages during this time (Table 1). The warm early Pleistocene CD 11 shift of Lagoe and Thompson (1988) in the Northern Pacific Ocean can be clearly recognized at Site 798 in the Japan Sea (Fig. 3). The mixed nature of the surface conditions in the sea during the late early Pleistocene and the late Pleistocene Brunhes Magnetochron is evident in the periodic $N$. pachyderma $C D$ shifts as well as the mixed nature of the foraminiferal assemblages. The CD shifts recorded in Brunhes sediments at Site 798 correlate well with the isotopic and faunal record of glaciation/deglaciation in the Northern Hemisphere and represent the most complete record of Pleistocene sea-surface temperature fluctuations in the Northern Pacific region analyzed to date (Figs. 3 and 4 and Table 3). However, the sea-surface temperature changes inferred from the CD shifts of $N$. pachyderma at Site 798 are not as firm in the early Brunhes, making the correlation to oxygen isotopic stages during this time period questionable. In this latter interval, several of the samples include significant abundances of warm-water species such as Globigerinoides ruber (Table 1); these samples are shown with arrows on Figure 4. Future, more detailed study and interpretation of the assemblages marked by the appearance of warm Tsushima taxa may change and enhance the present Brunhes sea-surface temperature record and therefore allow better correlation of the isotopic stage boundaries for the early Brunhes.

Six intervals dominated by dextrally coiling specimens of Neogloboquadrina pachyderma span the $0.73-\mathrm{m}$.y. period represented by the Brunhes Magnetochron and suggest periodic variations of the seasurface temperature at Site 798. Late Pleistocene sea-surface temperature fluctuations in the Northern Pacific and Atlantic oceans are related to orbital-scaled responses of ice volume associated with glaciation/deglaciation cycles. In the Atlantic Ocean orbitally scaled sea-surface temperature signals dominate at 41 and 100 k.y. (Ruddiman and McIntyre, 1984), whereas in the Northern Pacific Ocean the 41-k.y. response is the most dominant component as primarily established through study of changes in abundance of the radiolarian 
Table 3. Comparison of estimated depths of oxygen isotopic stage boundaries established at Site 798 based on Neogloboquadrina pachyderma coiling dominance with datums from shipboard studies.

\begin{tabular}{|c|c|c|}
\hline $\begin{array}{l}\text { Estimated depth } \\
\text { (mbsf) } \\
\text { (this study) }\end{array}$ & $\begin{array}{l}\text { Oxygen isotopic } \\
\text { stage boundary (age, ka) } \\
\text { (Imbric et al.. 1984: } \\
\text { Prell et al., 1986) }\end{array}$ & $\begin{array}{l}\text { Shipboard datum (age, ka) } \\
\text { (Ingle, Suyehiro, von Breymann, et al., 1990) }\end{array}$ \\
\hline 2.0 & $2 / 3(0.024)$ & \\
\hline 5.6 & $3 / 4(0.059)$ & \\
\hline 9.5 & $4 / 5(0.071)$ & \\
\hline 11.6 & $5 / 6(0.128)$ & \\
\hline 27.5 & $6.7(0.186)$ & \\
\hline 37.5 & $7 / 8(0.245)$ & \\
\hline 40.8 & & Last-appearance datum Rhizosolenia curvirostris $(0.27)$ \\
\hline 42.3 & $8.4(0.269)$ & \\
\hline 46.8 & $8 / 9(0.303)$ & \\
\hline 48.0 & $9 / 10(0.339)$ & \\
\hline 50.3 & $10 / 11(0.362)$ & \\
\hline 51.5 & $11 / 12(0.423)$ & \\
\hline-56.0 & & Last-appearance datum Pseudoemiliania lacunosa $(0.46)$ \\
\hline 57.3 & $12 / 13(0.478)$ & \\
\hline 62.5 & $13 / 14(0.524)$ & \\
\hline 66.6 & $14 / 15(0.565)$ & \\
\hline 70.4 & $15 / 16(0.62)$ & \\
\hline 73.4 & $16 / 17(0.659)$ & \\
\hline 75.3 & $17 / 18(0.689)$ & \\
\hline 77.9 & $18 / 19(0.726)$ & \\
\hline 80.1 & $19 / 20(0.736)$ & \\
\hline 80.3 & & Brunhes/Matuyama boundary ( 0.73$)$ \\
\hline 93.0 & & Matuyama/Jaramillo boundary $(0.91)$ \\
\hline 93.7 & & Jaramillo/Matuyama boundary $(0.98)$ \\
\hline-118.7 & & Last-appearance datum Helicosphaera sellii (1.2) \\
\hline 194.0 & & Matuyama/Olduvai boundary (1.66) \\
\hline
\end{tabular}

Theocalyptra davisiana (= Cycladophora davisiana) (Pisias and Leinen, 1984; Morley, 1987). Preliminary shipboard analyses of Pleistocene cyclic sequences at Site 798 suggest strong cyclicity at 41 k.y. (Ingle, Suyehiro, von Breymann, et al., 1990). Moreover, Föllmi et al. (this volume) demonstrated that an array of cyclicities at about $104,41.4,27,19,16,13$, and 10.5 k.y. can be identified at this site. The record of sea-surface temperature fluctuations based on the planktonic foraminifers does suggest the presence of similar cyclicities and is worthy of more detailed investigation.

Correlation of the estimated variations in sea-surface temperature at Site 798 with global oxygen isotopic stages (Imbrie et al., 1984; Prell et al., 1986) supplements the sediment-accumulation rates previously reported for the Brunhes Magnetochron (Ingle, Suyehiro, von Breymann, et al., 1990). Only three magnetostratigraphic and biostratigraphic datums are available for this period: (1) Brunhes/ Matuyama boundary at $0.73 \mathrm{Ma}$, (2) last-appearance datum of the nannofossil Pseudoemiliania lacunosa at $0.46 \mathrm{Ma}$, and (3) last-appearance datum of the diatom Rhizosolenia curvirostris at $0.27 \mathrm{Ma}$. Based on these three datums the average accumulation rate in the early Brunhes was much slower $(8.6 \mathrm{~cm} / \mathrm{k}$.y. $)$ than in the late Brunhes $(15 \mathrm{~cm} / \mathrm{k} . \mathrm{y}$.). Figure 5 shows a revised sediment-accumulation curve for the Brunhes at Site 798 based on the correlation of coiling dominance shifts of $N$. pachyderma with global oxygen isotope stages. According to this curve sediment accumulation during the early and late Brunhes increased from 7.7 to $16.1 \mathrm{~cm} / \mathrm{k} . \mathrm{y}$., which roughly confirms and supports the rates reported earlier. However, the numerous datum points detail the character of sediment-accumulation rate fluctuations in the early and late Brunhes and attest to the complexity of the depositional history at Site 798.

\section{CONCLUSIONS}

Quantitative analysis of Pleistocene planktonic foraminifers from Site 798 in the Japan Sea reveals the following:

1. More than $40 \%$ of the Pleistocene assemblages in the Japan Sea are dominated by sinistrally coiling specimens of Neogloboquadrina pachyderma representing polar-subpolar water masses and surface temperatures lower than $10^{\circ} \mathrm{C}$. Moreover, the sinistrally coiling as-

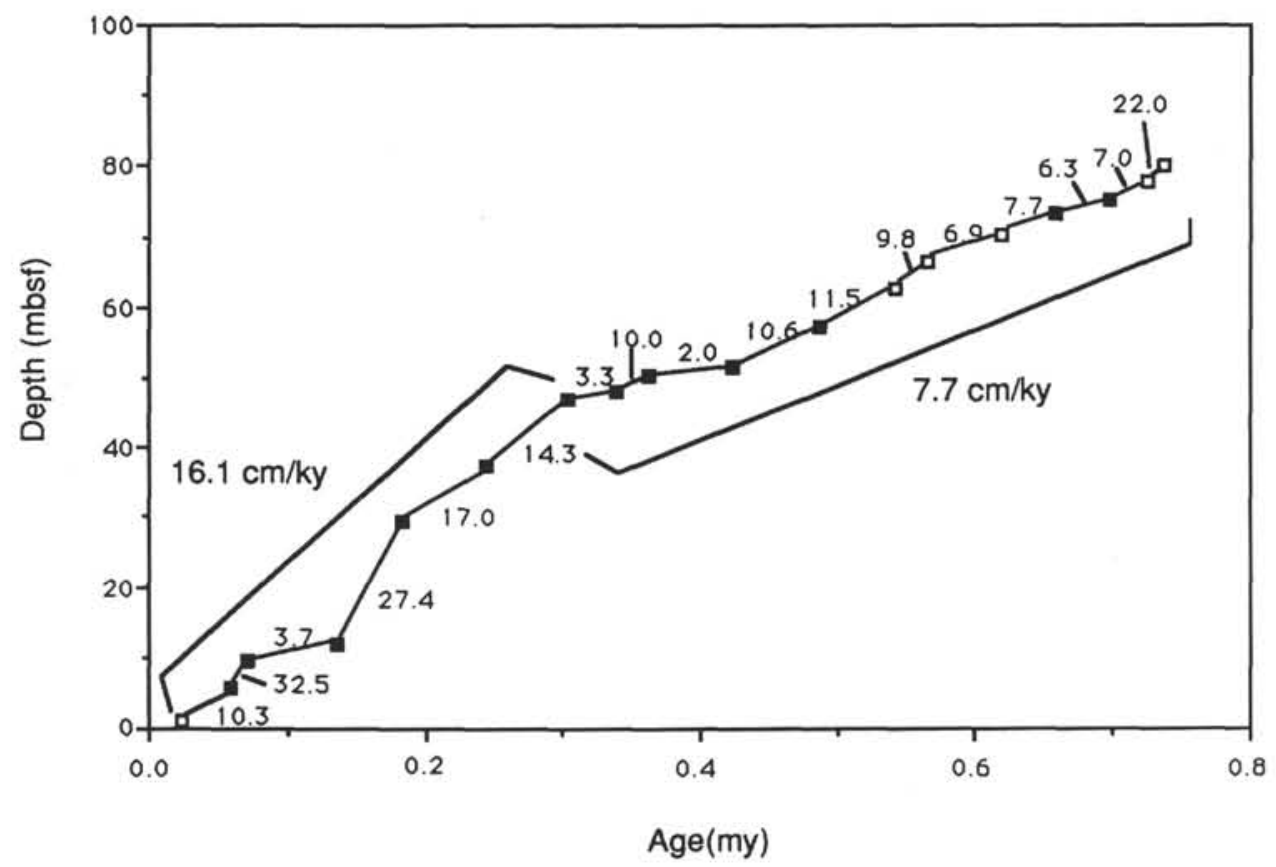

Figure 5. Sediment-accumulation rates for the Brunhes Magnetochron at Site 798 based on datums established from coiling dominance changes of Neogloboquadrina pachyderma (see Table 3). The open symbols represent questionable datum points. 
semblages are more common in late Pleistocene Brunhes time attesting to the restriction of the warm Tsushima Current into the Japan Sea during glacial lowstands of sea level.

2. Approximately $19 \%$ of the Pleistocene planktonic foraminiferal assemblages are rich in Globigerina bulloides, a species representative of upwelling conditions. These assemblages resemble the gyre-margin fauna in the adjacent Pacific Ocean and are most abundant during early Pleistocene time between 1.6 and $1.3 \mathrm{Ma}$ and during the late Pleistocene between 0.4 and $0.18 \mathrm{Ma}$. The occurrence of $G$. bulloides likely represents upwelling and increased productivity as a result of interaction between the warm Tsushima Current and the cold Liman Current during these periods.

3. Transitional assemblages dominated by dextrally coiling $\mathrm{NeO}$ globoquadrina pachyderma and Globorotalia inflata constitute about $16.5 \%$ of the assemblages analyzed at Site 798 and represent warm surface temperatures between $10^{\circ}$ and $18^{\circ} \mathrm{C}$. These assemblages are most abundant in the lower Pleistocene and suggest unrestricted flow of the Tsushima Current into the sea prior to onset of mid-latitude glaciation at $1.2 \mathrm{Ma}$.

4. Beginning at approximately $0.3 \mathrm{Ma}$ a small number of the samples (about $2.5 \%$ ) contains assemblages dominated by Globigerina quinqueloba and Globigerinita spp. The occurrence of these delicate species suggests excellent preservation possibly related to high production and flux rate for these taxa.

5. About $19 \%$ of the samples studied included fewer than 50 specimens of planktonic foraminifers or were completely barren. These samples are interpreted as evidence of severe dissolution. The occurrence of dissolved samples in Core 128-798A-12H suggests the onset of mid-latitude glaciation in the Northern Hemisphere.

6. Patterns of the coiling dominance of Neogloboquadrina pachyderma at Site 798 allow correlation with the Pleistocene sequence exposed on the Oga Peninsula of Honshu, as well as with open-ocean CD shifts established for the Northern Pacific Ocean by Lagoe and Thompson (1988). The warm early Pleistocene CD 11 shift of Lagoe and Thompson (1988) is clearly expressed at Site 798, suggesting unrestricted flow of the Tsushima Current into the Japan Sea at this time. Numerous warm/cold events expressed by faunal variations in the late early and late Pleistocene at Site 798 attest to fluctuating surface conditions (i.e., periodic isolation of the Japan Sea during low sea-level stands associated with glacial maxima).

7. The Brunhes record of sea-surface temperature fluctuations based on the coiling dominance of Neogloboquadrina pachyderma at Site 798 can be correlated to global Pleistocene isotopic stages established in the Northern Hemisphere. Correlation with oxygen isotopic stages 3-12 and 17 appears firm, whereas evidence and correlation to stages $2,13-16,18$, and 19 are tentative.

8. Previously reported sediment-accumulation rates for the Brunhes Magnetochron at Site 798 compare well to the rates calculated based on correlations of coiling dominance shifts of Neogloboquadrina pachyderma and global oxygen isotopic stages. According to these correlations the sediment-accumulation record is extremely complex at Site 798.

\section{ACKNOWLEDGMENTS}

I would like to thank my thesis advisor Dr. James C. Ingle, Jr., for making my participation on Leg 128 possible, continued support throughout this project, and several critical readings of this manuscript. I am grateful to Drs. Simon Brassell, Marie-Pierre Aubry, Charlotte Brunner, Robert Dunbar, and Lloyd Burckle for their advice and encouragement. I am deeply grateful to my mother, Vasti, for her continuous support throughout my graduate studies at Stanford. I am especially indebted to Charlotte Brunner for comments on an earlier draft of this paper. This project was funded through JOI-USSAC/TAMRF.

\section{REFERENCES}

Arikawa, R., 1983. Distribution and taxonomy of Globigerina pachyderma (Ehrenberg) off the Sanriku Coast, Northeast Honshu, Japan. Sci. Rep. Tohoku Univ., Ser. 2, 53:103-157.

Bandy, O. L., 1959. Geological significance of coiling ratios in the foraminifer Globigerina pachyderma (Ehrenberg). Geol. Soc. Am. Bull., 70:1708.

, 1960. The geological significance of coiling ratios in the foraminifer Globigerina pachyderma (Ehrenberg). J. Paleontol., 34:671-681.

Bé, A. W., and Thoderlund, D. S., 1971. Distribution and ecology of living planktonic foraminifera in surface waters of the Atlantic and Indian oceans. In Funnel, B. M., and Riedel, W. R. (Eds.), Micropaleontology of the Oceans: London (Cambridge Univ. Press), 105-149.

Berger, W. H., 1968. Planktonic foraminifera: selective solution and paleoclimatic interpretation. Deep-Sea Res. Part A, 15:31-34.

Bradshaw, J. S., 1959. Ecology of living planktonic foraminifera in the North and Equatorial Pacific Ocean. Contrib. Cushman Found. Foraminiferal Res., 10:25-64.

Broecker, W. S., and Denton, G. H., 1989. The role of ocean-atmosphere reorganization in glacial cycles. Geochim. Cosmochim. Acta, 53:2465-2501.

Butt, A., 1980. Biostratigraphic and paleoenvironmental analyses of the sediments at the Emperor Seamounts, DSDP Leg 55, Northwestern Pacific: Cenozoic foraminifers. In Jackson, E. D., and Koizumi, I., et al., Init. Repts. DSDP, 55: Washington (U.S. Govt. Printing Office), 289-325.

Chinzei, K., Fujioka, K., Kitazato, H., Koizumi, I., Oba, T., Oda, M., Okada, H., Sakai, T., and Tanimura, Y., 1987. Postglacial environmental change of the Pacific Ocean off the coast of central Japan. Mar. Micropaleontol., 11:273-291.

Cifelli, R., 1961. Globigerina incompta, a new species of pelagic foraminifera from the North Atlantic. Contrib. Cushman Found. Foram. Res., 12:83-86.

, 1973. Observations on Globigerina pachyderma (Ehrenberg) and Globigerina incompta Cifelli from the North Atlantic. J. Foraminiferal Res., 3:157-166.

Echols, R. J., 1973. Foraminifera, Leg 19, Deep Sea Drilling Project. In Creager, J. S., Scholl, D. W., et al., Init. Repts. DSDP, 19: Washington (U.S. Govt. Printing Office), 721-733.

Ericson, D. B., 1959. Coiling direction of Globigerina pachyderma as a climatic index. Science, 130:219-220.

Imbrie, J., Hays, J. D., Martinson, D. G., McIntyre, A., Mix, A. C., Morley, J. J., Pisias, N. G., Prell, W. L., and Shackleton, N. J., 1984. The orbital theory of Pleistocene climate: support from a revised chronology of the marine $\delta^{18} \mathrm{O}$ record. In Berger, A. L., Imbrie, J., Hays, J., Kukla, G., and Saltzman, B. (Eds.), Milankovitch and Climate (Pt. 2): Boston (D. Reidel), 269-305.

Imbrie, J., and Kipp, N. G., 1971. A new micropaleontological method for quantitative paleoclimatology: application to a late Pleistocene Caribbean core. In Turekian, K. K. (Ed.), The Late Cenozoic Glacial Ages: New Haven (Yale Univ. Press), 71-181.

Ingle, J. C., Jr., 1973a. Neogene foraminifera from the Northeastern Pacific Ocean, Leg 18 Deep Sea Drilling Project. In Kulm, L. D., von Huene, R., et al., Init. Repts. DSDP, 18: Washington (U.S. Govt. Printing Office), 517-567.

- 1973b. Summary comments on Neogene biostratigraphy, physical stratigraphy, and paleo-oceanography in the marginal Northeastern Pacific Ocean. In Kulm, L. D., von Huene, R., et al., Init. Repts. DSDP, 18: Washington (U.S. Govt. Printing Office), 949-960.

, 1975. Pleistocene and Pliocene foraminifera from the Sea of Japan, Leg 31 Deep Sea Drilling Project. In Karig, D. E., and Ingle, J. C., et al., Init. Repts. DSDP, 31: Washington (U.S. Govt. Printing Office), 693-701.

Ingle, J. C., Jr., Suyehiro, K., von Breymann, M. T., et al., 1990. Proc. ODP, Init. Repts., 128: College Station, TX (Ocean Drilling Program).

Karig, D. E., Ingle, J. C., Jr., et al., 1975. Init. Repts. DSDP, 31: Washington (U.S. Govt. Printing Office).

Keller, G., 1978. Morphologic variation of Neogloboquadrina pachyderma (Ehrenberg) in sediments of the marginal and central Northeast Pacific Ocean and paleoclimatic interpretation. J. Foraminiferal Res., 8:208-224. 1980. Planktonic foraminiferal biostratigraphy and paleoceanography of the Japan Trench, Leg 57, Deep Sea Drilling Project. In von Huene, R., Nasu, N., et al., Init. Repts. DSDP, 56, 57 (Pt. 2): Washington (U.S. Govt. Printing Office), 809-833.

Kennett, J. P., and Srinivasan, M. S., 1980. Surface ultrastructural variation in Neogloboquadrina pachyderma (Ehrenberg): phenotypic variation 
and phylogeny in the late Cenozoic. Cushman Found. Spec. Publ., 19:134-162.

Kent, D., Opdyke, N. D., and Ewing, M., 1971. Climatic change in the North Pacific using ice-rafted detritus as a climatic indicator. Geol. Soc. Am. Bull., 82:2741-2754.

Kipp, N. G., 1976. New transfer function for estimating past sea-surface conditions from sea-bed distribution of planktonic foraminiferal assemblages in the North Atlantic. In Cline, R. M., and Hayes, J. D. (Eds.), Investigations of Late Quaternary Paleoceanography and Paleoclimatology. Mem.-Geol. Soc. Am., 145:3-41.

Lagoe, M. B., 1986. Foraminifers from the Nankai Trough and the Japan Trench. In Kagami, H., Karig, D. E., and Coulbourn, W. C., et al., Init. Repts. DSDP, 87: Washington (U.S. Govt. Printing Office), 587-603.

Lagoe, M. B., and Thompson, P. R., 1988. Chronostratigraphic significance of late Cenozoic planktonic foraminifera from the Ventura Basin, California: potential for improving tectonic and depositional interpretation. $J$. Foraminiferal Res., 18:250-266.

Maiya, S., 1975. Late Cenozoic planktonic foraminiferal biostratigraphy of the oil-field region of Northeast Japan. Jpn. Pet. Explor. Co., Tech. Lab. Spec. Rep., 35:1-97.

1978. Late Cenozoic planktonic foraminiferal biostratigraphy of the oil-field region of northeast Japan. In Cenozoic Geology of Japan, Osaka, 35-60. (English abstract)

Maiya, S., Saito, T., and Sato, T., 1976. Late Cenozoic planktonic foraminiferal biostratigraphy of Northwest Pacific sedimentary sequences. In Takayanagi, Y., and Saito, T. (Eds.), Progress in Micropaleontology: New York (Micropaleontology Press), 395-422.

Matoba, Y., 1984. Paleoenvironment of the Sea of Japan. In Oertli, H. J. (Ed.), Benthos '83: 2nd Int. Symp. Benthic Foraminifera, 409-414.

Matoba, Y., Tomizawa, A., Maruyama, T., and Fukasawa, K., 1990. Neogene and Quaternary sedimentary sequences in the Oga Peninsula. Benthos '90: 4th Int. Symp. on Benthic Foraminifera, B1-64.

Meyers, P. A., Takemura, K,. and Horie, S., in press. Quaternary climate history of Japan: a synthesis of the sedimentary records from Lake Biwa, the Sea of Japan, and the western Pacific. Quat. Res.

Morley, J. J., 1987. Late Pleistocene time series of atmospheric and oceanic variables recorded in sediments from the subarctic Pacific. Paleoceanography, 2:49-62.

Oba, T., Kato, M., Kitazato, H., Koizumi, I., Omura, A., Sakai, T., and Takayama, T., 1991. Paleoenvironmental changes in the Japan Sea during the last 85,000 years. Paleoceanography, 6:499-518.

Olsson, R. K., 1974. Pleistocene paleoceanography and Globigerina pachyderma (Ehrenberg) in Site 36, DSDP, Northeastern Pacific. J. Foraminiferal Res., 4:47-60.

1976. Wall structure, topography and crust of Globigerina pachyderma (Ehrenberg). In Takayanagi, Y., and Saito, T. (Eds.), Prog. ress in Micropaleontology: New York (Micropaleontology Press), 244257.

Olsson, R. K., and Goll, R., 1970. Cruise leg synthesis, biostratigraphy, In McManus, D. A., Burns, R. E., et al., Init. Repts. DSDP, 5: Washington (U.S. Govt. Printing Office), 809-833.

Parker, F. L., and Berger, W. H., 1971. Faunal and solution patterns of planktonic foraminifera in surface sediments of the South Pacific. DeepSea Res. Part A, 18:73-107.
Pisias, N. G., and Leinen, M., 1984. Milankovitch forcing of the oceanic system: evidence from the Northwest Pacific. In Berger, A. L., Imbrie, J., Hays, J., Kukla, G., and Saltzman, B. (Eds.), Milankovitch and Climate (Pt. 1): Boston (D. Reidel), 307-330.

Prell, W. L., Imbrie, J., Martinson, D. G., Morley, J. J., Pisias, N. G., Shackleton, N. J., and Streeter, H. F., 1986. Graphic correlation of oxygen isotope stratigraphy application to the late Quaternary. Paleoceanography, 1:137-162.

Reynolds, L., and Thunell, R. C., 1985. Seasonal succession of planktonic foraminifera in the subpolar North Pacific. J. Foraminiferal Res., 15:282-301.

Rohling, E., and Gieskes, W.C., 1989. Late Quaternary changes in Mediterranean intermediate water density and formation rate. Paleoceanography, 4:531-546.

Ruddiman, W. F., and McIntyre, A., 1984. Ice-age thermal response and climatic role of the surface North Atlantic Ocean, $40^{\circ} \mathrm{N}$ to $63^{\circ} \mathrm{N}$. Geol. Soc. Am. Bull., 95:381-396.

Sautter, L., and Thunell, R. C., 1989. Seasonal succession of planktonic foraminifera: results from a four-year time-series sediment trap experiment in the northeast Pacific. J. Foraminiferal Res., 19:253-267.

Savin, S. M., 1977. The history of the Earth's surface temperature during the past 100 million years. Annu. Rev. Earth Planet. Sci. Lett., 5:319-355.

Shackleton, N. J., and Opdyke, N. D., 1977. Oxygen isotope and paleomagnetic evidence for early Northern Hemisphere glaciation. Nature, 270:216-219.

Tamaki, K., 1986. Age estimation of the Japan Sea on the basis of stratigraphy, basement depth and heat flow data. J. Geomagn. Geoelectr., 38:427-446.

Tamaki, K., Pisciotto, K., Allan, J., et al., 1990. Proc. ODP, Init. Repts., 127: College Station, TX (Ocean Drilling Program).

Thompson, P. R., 1980. Foraminifers from Deep Sea Drilling Project Sites 434, 435, and 436, Japan Trench: DSDP Leg 56. In von Huene, R., Nasu, N., et al., Init. Repts. DSDP. 56, 57 (Pt. 2): Washington (U.S. Govt. Printing Office), 775-807.

, 1981. Planktonic foraminifera in the western North Pacific during the past 150,000 years: comparison of modern and fossil assemblages. Palaeogr., Palaeoclimatol., Palaeoecol., 35:241-279.

Thompson, P. R., and Shackleton, N. J., 1980. North Pacific paleoceanography: late Quaternary coiling variations of planktonic foraminifer Neogloboquadrina pachyderma. Nature, 287:829-833.

Thunell, R. C., and Honjo, S., 1981. Planktonic foraminiferal flux to the deep ocean: sediment trap results from the tropical Atlantic and the central Pacific. Mor. Geol., 40:237-253.

Ujiie, J., and Ichikura, M., 1973. Holocene to uppermost Pleistocene planktonic foraminifers in a piston core from off the San'in district, Sea of Japan. Trans. Paleontol. Soc. Jpn., 91:137-150.

Vincent, E., 1975. Neogene planktonic foraminifera from the central North Pacific, Leg 32, Deep Sea Drilling Project. In Larson, R. L., Moberley, R., et al., Init. Repts. DSDP, 32: Washington (U.S. Govt. Printing Office), 765-801.

Date of initial receipt: 19 April 1991

Date of acceptance: 28 February 1992

Ms 127/128B-149 